\title{
MIMO Channel Modelling
}

\author{
Faisal Darbari, Robert W. Stewart and Ian A. Glover \\ University of Strathclyde, Glasgow \\ United Kingdom
}

\section{Introduction}

Multiple antenna communications technologies offer significant advantages over single antenna systems. These advantages include extended range, improved reliability in fading environments and higher data throughputs. If multiple antennas are provided only at the transmitting end of a link then the system is referred to as multiple input single output (MISO). If multiple antennas are provided only at the receiving end of a link then the system is referred to as single input multiple output (SIMO). If multiple antennas are provided at both ends of a link then the system is referred to as multiple input multiple output (MIMO).

Multiple antenna systems can be divided into two classes depending on the signal processing employed. These are: (i) smart antennas and (ii) spatial multiplexors.

Smart antennas provide increased signal-to-noise-and-interference ratio (SNIR) via diversity gain, array gain and/or interference suppression. Each transmit antenna radiates, to within a simple gain and delay difference, the same signal. Similarly, each receive antenna contributes its signal to a gain and delay weighted sum. By setting transmit and receive gains and delays appropriately, improved SNIR is achieved which may be used to realise greater spectral efficiency (and, therefore, greater channel capacity), greater range and/or decreased latency (due to a reduced requirement for channel coding).

Spatial multiplexors can provide increased channel capacity directly. Each transmit antenna radiates an independent signal sub-stream. With $N$ transmit and $N$ receive antennas, for example, an $\mathrm{N}$-fold increase in data-rate is possible (in principle) over that achievable with a single input single output (SISO) antenna system (without any increase in total transmitted power).

In this chapter a theoretical framework for describing MIMO channels is presented followed by a brief outline of MIMO channel modelling principles [P. Almer et. al., 2007]. The rest of the chapter describes a selection of widely adopted MIMO channel models, their capabilities and limitations, in the context of specific standards. 


\section{MIMO channel framework}

Wireless channels are linear. They may, therefore, be represented by a linear filter and described by their impulse response. If the transmit and receive antennas, and the scattering objects in the environment, are static then the channel will be time-invariant and the impulse response, $h(\tau)$, will be a function of delay, $\tau$, only. If the transmit antenna, receive antenna or scattering objects move then the channel will be time-variant and the impulse response, $h(t, \tau)$, will be a function of time, $t$.

From a communication systems point of view $h(t, \tau)$ is an entirely adequate channel description, i.e. it represents sufficient information to predict the (noiseless) received signal from the transmitted signal. It has the limitation, however, of obscuring the underlying propagation physics. If $h(t, \tau)$ is to be predicted from full or partial knowledge of the physical environment, then models linking it to the most important aspect of the environment, i.e. the spatial distribution of scattering objects, is required. The doubledirectional impulse response is a channel description that makes explicit this connection between the systems-level impulse response and propagation physics.

\subsection{Double-directional impulse response}

The impulse response of the wireless transmission channel describes the cascaded effect of transmit antenna, propagation channel and receive antenna. Changing an antenna, therefore, may change the impulse response, even though the propagation channel (i.e. the physical arrangement of scatterers) may remain the same. The effects on $h(t, \tau)$ of the antennas and propagation environment can be decoupled using a description of the channel called the double-directional impulse response [M. Steinbauer et. al., 2001], i.e.:

$$
h(t, \tau)=\int_{4 \pi 4 \pi} \int_{4} g_{T}\left(\boldsymbol{\varphi}_{T}\right) p\left(t, \tau, \boldsymbol{\varphi}_{R}, \boldsymbol{\varphi}_{T}\right) g_{R}\left(\boldsymbol{\varphi}_{R}\right) d \boldsymbol{\varphi}_{T} d \boldsymbol{\varphi}_{R}
$$

where the integrand is the component of impulse response represented by power leaving the transmit antenna with (vector) direction $\boldsymbol{\varphi}_{T}$ (or, more strictly speaking, within the element of solid angle, $d \boldsymbol{\varphi}_{T}$, centred on $\left.\boldsymbol{\varphi}_{T}\right)$ and arriving at the receive antenna with (vector) direction $\varphi_{R} \cdot \mathrm{g}_{R}\left(\boldsymbol{\varphi}_{R}\right)$ and $\mathrm{g}_{T}\left(\boldsymbol{\varphi}_{T}\right)$ are the complex (field-strength or voltage) gains of the receive and transmit antennas, respectively, in the directions $\varphi_{R}$ and $\varphi_{T}$. (The magnitude of the voltage gain is the square root of the conventional antenna (power) gain and is proportional to an antenna's effective length - the square root of its effective area - via the antenna reciprocity formula.) For isotropic antennas $g_{R}\left(\varphi_{R}\right)=g_{T}\left(\varphi_{T}\right)=1 . p\left(t, \tau, \varphi_{R}, \varphi_{T}\right)$ is the component of impulse response assuming isotropic antennas. It incorporates all propagation related losses including free-space path loss, absorption and scattering loss. The integral in Eq. (1) sums over all possible directions-of-departure (DODs) at the transmitter and all possible directions-of-arrival (DOAs) at the receiver.

If propagation is via $K$ discrete paths then Eq. (1) can be written as a summation over $K$ multipath components (MPCs), i.e.: 


$$
h(t, \tau)=\sum_{i=1}^{K} g_{T}\left(\boldsymbol{\varphi}_{T_{i}}\right) p\left(t, \tau, \boldsymbol{\varphi}_{R_{i}}, \boldsymbol{\varphi}_{T_{i}}\right) g_{T}\left(\boldsymbol{\varphi}_{T_{i}}\right)
$$

Recognising that the channel characteristics are a function of transmit and receive antenna locations, denoted by the position vectors $\mathbf{r}_{T}$ and $\mathbf{r}_{R}$, respectively then the double-directional impulse response may be more fully expressed using $p\left(\mathbf{r}_{T}, \mathbf{r}_{R}, t, \tau, \boldsymbol{\varphi}_{R}, \boldsymbol{\varphi}_{T}\right)$, i.e.:

$$
h\left(\mathbf{r}_{T}, \mathbf{r}_{R}, t, \tau\right)=\sum_{i=1}^{K} g_{T}\left(\boldsymbol{\varphi}_{T_{i}}\right) p\left(\mathbf{r}_{T}, \mathbf{r}_{R}, t, \tau, \boldsymbol{\varphi}_{R_{i}}, \boldsymbol{\varphi}_{T_{i}}\right) g_{T}\left(\boldsymbol{\varphi}_{T_{i}}\right)
$$

The description above relates to singly-polarised antennas. A more complete channel description can be devised by introducing a matrix of impulse responses defining the coupling between, for example, the vertically- and horizontally-polarised ports of a dualpolarised transmit antenna and the vertically- and horizontally-polarised ports of a dualpolarised receive antenna, i.e.:

$$
\mathbf{h}(t, \tau)=\left[\begin{array}{ll}
h_{v v}(t, \tau) & h_{v h}(t, \tau) \\
h_{h v}(t, \tau) & h_{h h}(t, \tau)
\end{array}\right]
$$

The leading diagonal (co-polar) elements of Eq. (4) describe coupling between the vertically polarised port of the transmit antenna and the vertically polarised port of the receive antenna $\left(h_{v v}\right)$, and the horizontally polarised port of the transmit antenna and the horizontally polarised port of the receive antenna $\left(h_{h h}\right)$. The off diagonal (cross-polar) elements describe coupling between the horizontally polarised port of the transmit antenna and the vertically polarised port of the receive antenna $\left(h_{v h}\right)$, and the vertically polarised port of the transmit antenna and the horizontally polarised port of the receive antenna $\left(h_{h v}\right)$. In principle, the ports could have any pair of orthogonal polarisations. In practice, however, they are almost always perpendicular linear polarisations (typically vertical and horizontal for terrestrial links) or counter-rotating (right-handed and left-handed) circular polarisations.

Eq. (4) is a systems description. To express the cross-polarising effects of the antenna and propagation medium separately a cascade of three polarisation matrices (one each for transmit antenna, medium and receive antenna) is required for each propagation path. The system matrix for a discrete set of propagation paths is then given by: 


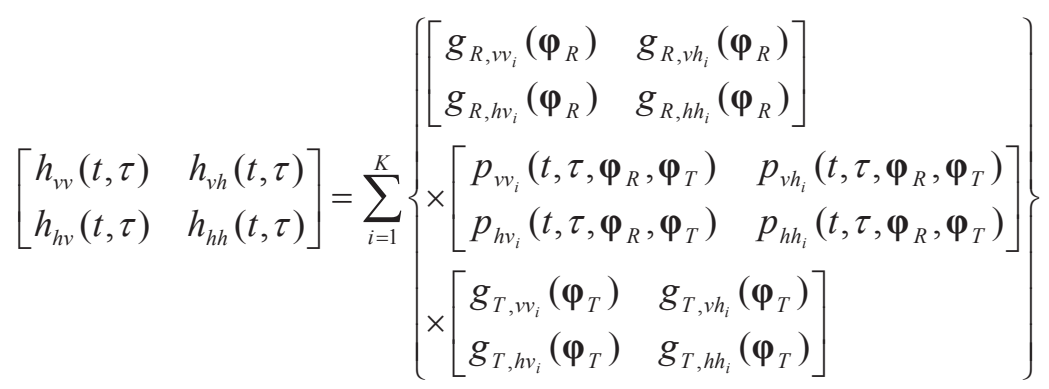

The use of vertical and horizontal basis polarisations (or, more generally, any pair of perpendicular linear polarisations) in Eq. (5) is problematic. A simple cartesian definition of a pair of basis polarisations (definition 1, Fig. 1) is adequate only for the single propagation path described by $\varphi_{R}=\varphi_{T}=0$. For propagation paths with non-zero DODs and DOAs some other pair of basis polarisations must be adopted [A. C. Ludwig., 1973]. The polarisations of a pair of dipoles (one electric, one magnetic) can be used - definition 2, Fig. 1 - as can the polarisations of a pair of perpendicular Huygens' sources - definition 3, Fig. 1. (A Huygens' source is the elementary radiating source of an electromagnetic wave referred to in Huygens ' Principle which states that each point on a propagating wave-front acts as a secondary source of radiation.)

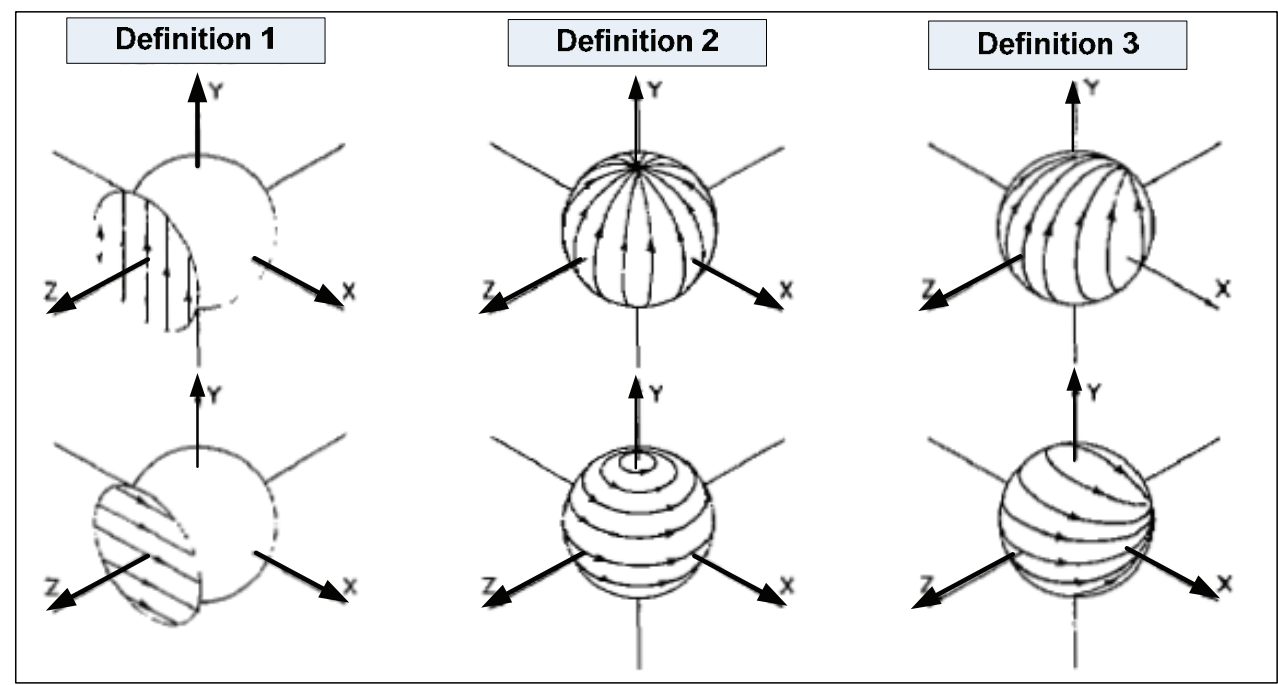

Fig. 1. Ludwig's three definitions of orthogonal basis polarisations (After [A. C. Ludwig, 1973]. (C 1973 IEEE))

Definitions 2 and 3 allow a pair of perpendicular polarisations to be defined for directions other than $\varphi_{R}=\varphi_{T}=0$ whilst preserving the requirement for transverse electromagnetic fields. They still suffer from limitations, however, since definition 2 does not define polarisation in the $\pm \mathbf{Y}$ direction and definition 3 does not define polarisation in the $-Z$ 
direction. (This is reflected physically by the fact that a dipole does not radiate along its axis - it has a toroidal radiation pattern - and a Huygens source does not radiate in the backward propagating direction - its radiation pattern is a cardioid of revolution.)

For radio links with columnated-beam antennas, and DODs and DOAs closely clustered about transmit and receive antenna boresights, the most appropriate choice of basis polarisations is probably definition 3. (As the spread DODs and DOAs about boresight tends to zero then all three definitions converge.) For links with omnidirectional antennas, and DODs and/or DOAs widely spread in azimuth (but not too widely spread in elevation), then definition 2 is probably more appropriate.

\subsection{The MIMO channel impulse response}

Fig. 2 shows a schematic diagram of a MIMO channel.

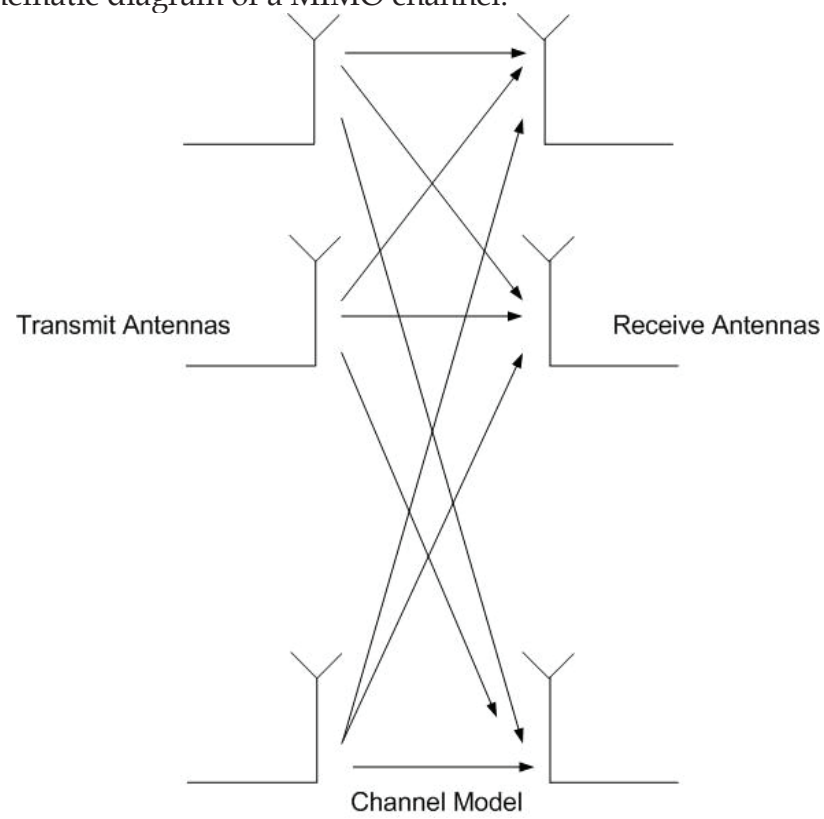

Fig. 2. MIMO channel

The MIMO channel must be described for all transmit and receive antenna pairs. For $M$ transmit antennas and $N$ receive antennas the MIMO transmission channel can be represented by an $N \times M$ channel matrix, i.e.: 


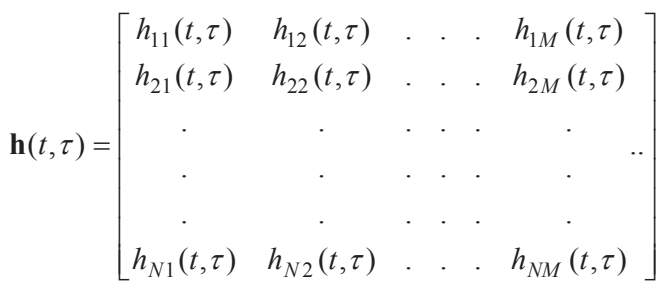

where $h_{n m}(t, \tau)$ represents the time-variant impulse response between the input of the $m^{\text {th }}$ transmit antenna and the output of the $n^{\text {th }}$ receive antenna. Each impulse response is the cascaded effect of transmit antenna, the propagation medium and a receive antenna. This is therefore a system-level representation. If polarisation diversity is employed then each element of the matrix must be replaced by the polarisation matrix of Eq. (4). This is equivalent to doubling the number of antennas at each end of the link; a dual-polarised antenna being treated, effectively, as two singly-polarised antennas. Any time variation, due to shadowing and/or multipath fading, arises from antenna motion or the motion of environmental scatterers. The spatial, polarisation and temporal correlations between the signals at the terminals of different receiving antennas are reflected in the correlation properties of the matrix elements.

MIMO channel models may be physical or analytical. Physical models are based either on a physical theory (often geometrical optics) or on physical measurements. They are sitespecific or specific to an environment type (e.g. urban suburban, rural) and are particularly useful in network planning. Analytical models are based on mathematical assumptions about channel behaviour. They are generally site-independent and are mostly used for system design, comparison and testing.

Physical models can be subdivided into deterministic and stochastic variants. Deterministic models are environment specific and are derived from the underlying physical radio propagation processes, e.g. reflection, diffraction, shadowing and wave-guiding. The most important example is ray tracing. Stochastic (physical) models are more generic than deterministic models. They are based on the fact that whilst, in the absence of a detailed environment database, the precise physical propagation parameters (e.g. DOD, DOA, number of paths, path delay, path power) are unpredictable, they nevertheless have welldefined statistical behaviours. Probability models can therefore be constructed for these propagation parameters. They are generally more computationally efficient than deterministic models. The Spatial Channel Model (SCM) and the Spatial Channel Model Extended (SCME) described in sections 4.1.1 and 4.1.2 are stochastic physical models.

Analytical channel models derive the MIMO channel matrix without any consideration of propagation parameters. Examples include Independent Identically Distributed (i.i.d.), Weichselberger and Kronecker models. The WiMAX and IEEE 802.11n channel models described in sections 4.2 and 4.3 are Kronecker models. Since MIMO channel matrices are easily generated using analytical models, and since the statistics of these matrices are both unvarying (repeatable) and environment independent, they are popular for the 
development, verification and optimisation of system hardware and software (especially signal processing algorithms).

\section{Link-level and system-level simulations}

Most standardised MIMO channel models provide link-level and system-level simulation. The former refers to a single point-to-point link (but including, of course, multiple transmit and receive antennas). The latter refers to multiple communication links, potentially including multiple base-stations (BSs). In the case of an SCM channel, for example, a calibration process is undertaken at the link-level prior to a system-level simulation.

The SCM and SCME system-level model defines a 'drop' concept where a mobile is placed (dropped) in a sequence of different network locations. The locations may be random or predefined by the user. Each drop represents a snapshot of the fading channel. The statistics of the channel parameters within a single drop are assumed to be stationary and, for the duration of the drop all large scale parameters (DOA, DOD, mobile station velocity etc.) are assumed to be constant. The drop concept is illustrated in Fig. 3.

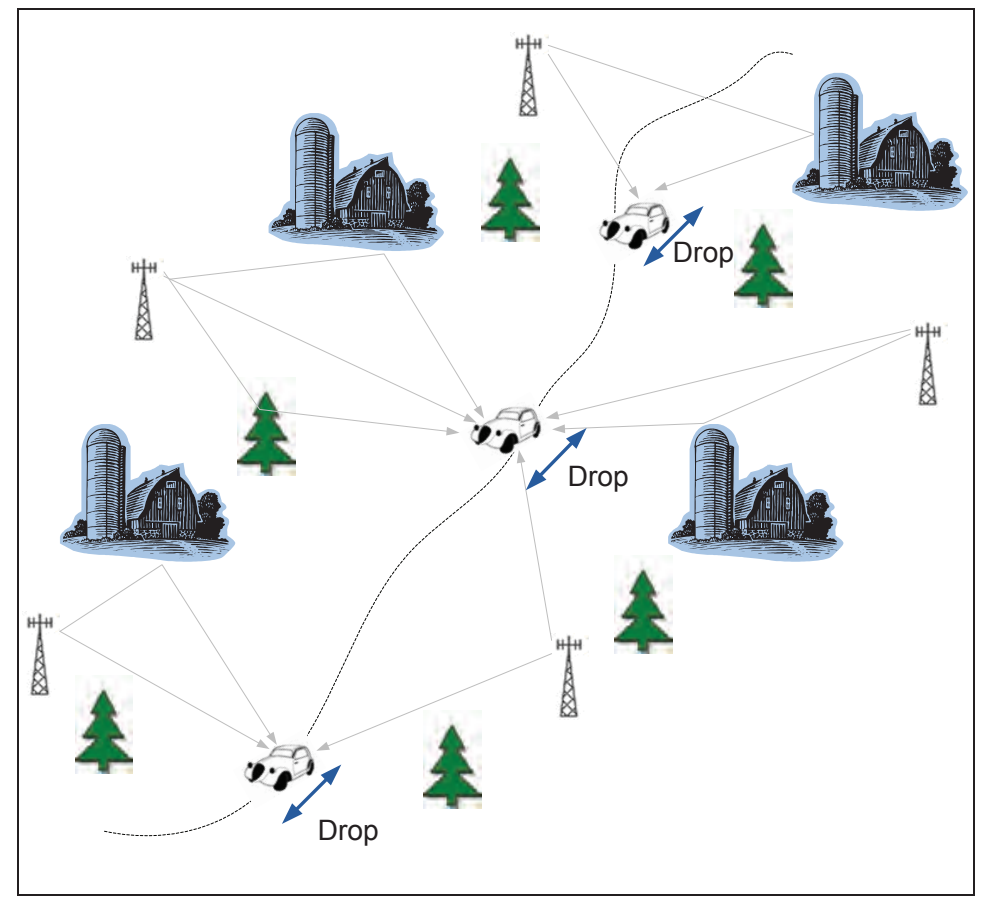

Fig. 3. Quasi-stationary drop periods in a channel simulation

A variation of the drop-based simulation allows the large-scale channel parameters to evolve (change gradually and continuously) at each simulation step inside a drop. This 
approach is more realistic than the stationary assumption but represents an increase in complexity and results in increased simulation time.

If motion of the mobile station (MS) and scatterers is assumed to be negligible during the course of a packet transmission the channel is said to be quasi-static. Such a channel has a decorrelation time, $T_{c}$, which is greater than the packet duration. If $T_{c}$ is defined as the time shift resulting in a correlation coefficient of 0.5 then it may be related (approximately) to the maximum Doppler shift, $f_{m}$ (speed/wavelength) [T. S. Rappaport., 2002], by:

$$
T_{c} \approx \frac{9}{16 \pi f_{m}}
$$

For a carrier frequency of $2 \mathrm{GHz}$ and a terminal speed of $3 \mathrm{~km} / \mathrm{h}$ the maximum Doppler shift would be $5.6 \mathrm{~Hz}$ and the channel decorrelation time would be $32 \mathrm{~ms}$. The assumption that the channel is quasi-static could then be justified providing the transmitted packet duration is less than this.

\section{Channel model standards}

Specific standardised channel models have been developed for the testing and optimisation of particular wireless standards in a manner that is repeatable and widely agreed. These wireless channel models are important for the development of new wireless devices. Examples of standardised channel models include COST 207, SCM and SCME. These are wideband power delay profile (PDP) models used in the development of GSM, WCDMA and LTE systems, respectively. Standardised channel models provide a framework to test algorithms and investigate design trade-offs thereby informing key design decisions relating to modulation, coding and multiple-accessing etc.

A selection of standardised channel models for mobile, broadband wireless access (BWA) and wireless local area network (WLAN) applications is given below:

\begin{tabular}{|l|l|l|}
\hline Channel Model & Origin & Application \\
\hline & & \\
\hline SCM & 3GPP/3GPP2 & 3G Outdoor \\
\hline SCME & WINNER & 3G Outdoor \\
\hline WIN II & WINNER II & 3G Outdoor \\
\hline SUI & Stanford University & Fixed BWA \\
\hline WiMAX- ITU-TDL & WiMAX form & Fixed/Mobile BWA \\
\hline IEEE 802.11n & IEEE 802.11n TGn (High throughput task group) & Indoor Channel \\
\hline
\end{tabular}

\subsection{WINNER}

The European WINNER (wireless world initiative new radio) project began in 2004 with the aim to develop a new radio concept for beyond third generation (B3G) wireless systems. Work Package 5 (WP5) of the WINNER projects focused on multi-dimensional channel 
modelling for carrier frequencies between 2 and $6 \mathrm{GHz}$ and bandwidths up to $100 \mathrm{MHz}$. In total six organisations were formally involved in WP5 (Elektrobit, Helsinki University of Technology, Nokia, Royal Institute of Technology (KTH), the Swiss Federal Institute of Technology (ETH) and the Technical University of IImenau.

At the start of the project there was no widely accepted channel model suitable for WINNER system modelling. Two existing channel models, 3GPP/3GPP2 Spatial Channel Model (SCM), were selected as starting points for outdoor simulation and one existing channel model (IEEE 802.11 TGn Model [3GPP TR25.996 V6.1.0, 2003-09]) was selected as a starting point for indoor simulation. The SCM had insufficient bandwidth and too few scenarios. In 2005 the first extension of SCM, SCM Extended (SCME), was therefore proposed. Despite the modifications to SCM, SCME was deemed inadequate for the simulation of B3G systems.

At the end of 2005 the WINNER channel model - Phase 1 (WIM1) was described in the deliverable D5.4 [WINNER, 2005]. WIM1 has a unified structure for indoor and outdoor environments and is based on double-directional measurement campaigns carried out in the $5 \mathrm{GHz}$ ISM2 band with bandwidths of up to $120 \mathrm{MHz}$. It covers six different propagation scenarios, i.e.(i) indoor small office, (ii) indoor hall, (iii) urban microcell, (iv) urban macrocell, (v) suburban macrocell, and (vi) rural . Both line-of-sight (LOS) and non-line-ofsight (NLOS) propagation conditions are catered for [H. El-Sallabi et. al., 2006].

In September 2007, the WINNER channel model - Phase II (WIM2) was described [WINNER II interim, 2006]. This model, which evolved from WIM1 and the WINNER II interim channel models, extended the propagation scenarios to: (i) indoor office, (ii) large indoor hall, (iii) indoor-to-outdoor, (iv) urban microcell, (v) bad urban microcell, (vi) outdoor-toindoor, (vii) stationary feeder, (viii) suburban macrocell, (ix) urban macrocell, (x) rural macrocell, and (xi) rural moving networks. In the course of the WINNER project channel models were implemented in MATLAB and made available through the official web site [WINNER II, 2007].

\subsubsection{Spatial Channel Model (SCM)}

SCM was developed by 3GPP/3GPP2 (third generation partnership project) for outdoor environments at a carrier frequency of $2 \mathrm{GHz}$. It was designed to test $5 \mathrm{MHz} \mathrm{CDMA}$ channels [P.Almer et. al., 2007] and consists of two parts: (i) a link level simulation model and (ii) a system-level simulation model. The SCM system level model is currently used as a de-facto standard for LTE, WCDMA, UMTS and WiMAX system level evaluation and performance verification.

\subsubsection{Link-level model}

The link-level model which might also be referred to as a reference model is a single link channel model. It provides a well-defined, convenient, interface for different equipment manufacturers to compare their proprietary implementations of the same signal processing algorithms. Link-level simulations alone are not recommended for performance testing of different algorithm because they reflect only a single snapshot of the (dynamic) channel. Link-level simulations do not, therefore, allow conclusions to be made about the general 
behaviour of a system and if such conclusions are required system-level simulations must be performed.

The link-level SCM can be implemented as either a stochastic physical or analytical model. In the former the wideband characteristics of the channel are modelled as a tapped delay line (TDL). Each tap is independently faded and is characterised by an azimuth DOD/DOA angular spectrum described by a uniform distribution (for MSs) or a Laplacian distribution (for BSs). The mean direction and angular spread at BS and MS are fixed (and thus represent stationary channel conditions). The Doppler spectrum is calculated based on the MS velocity (speed and direction relative to the line connecting MS and BS). The model also defines the number and configuration of antennas at MS and BS. Given all these parameters the physical model can be transformed to an analytical model [P.Almer et. al., 2007].

\subsubsection{System-level model}

The system-level model is a multi-link physical model intended for performance evaluation in which each link represents a cell or a sector within a cell. Fig. 4 illustrates a system-level simulation in which an MS receives interference from adjacent sectors of adjacent cells.

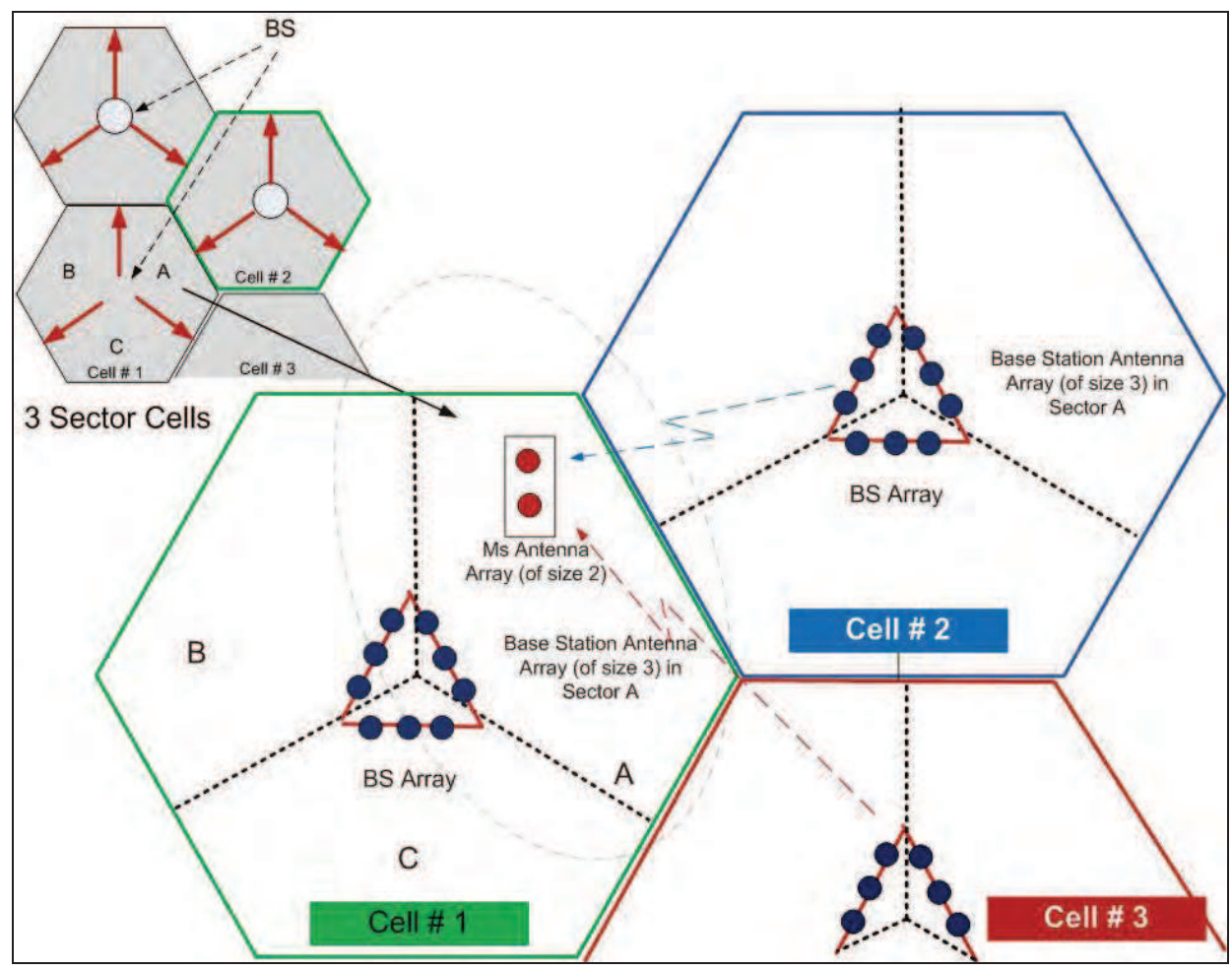

Fig. 4. SCM system level simulation. 
Each link comprises an MS and BS MIMO antenna array. Propagation is via multipaths and sub-paths. The excess delays of sub-paths are closely clustered around the delay of their (parent) multipath. This is assumed to originate from an environment with closely spaced clusters of scatterers. Fig. 5 illustrates the clustered scatterers and resulting multipaths and sub-paths.

The SCM distinguishes between three different environments, i.e. (i) urban macrocell, (ii) suburban macrocell and (iii) urban microcell. The modelling and simulation methodology are identical for all three environments but the parameters (e.g. azimuth spread, delayspread, shadow fading and path loss) are different [P. Almer et. al., 2007].

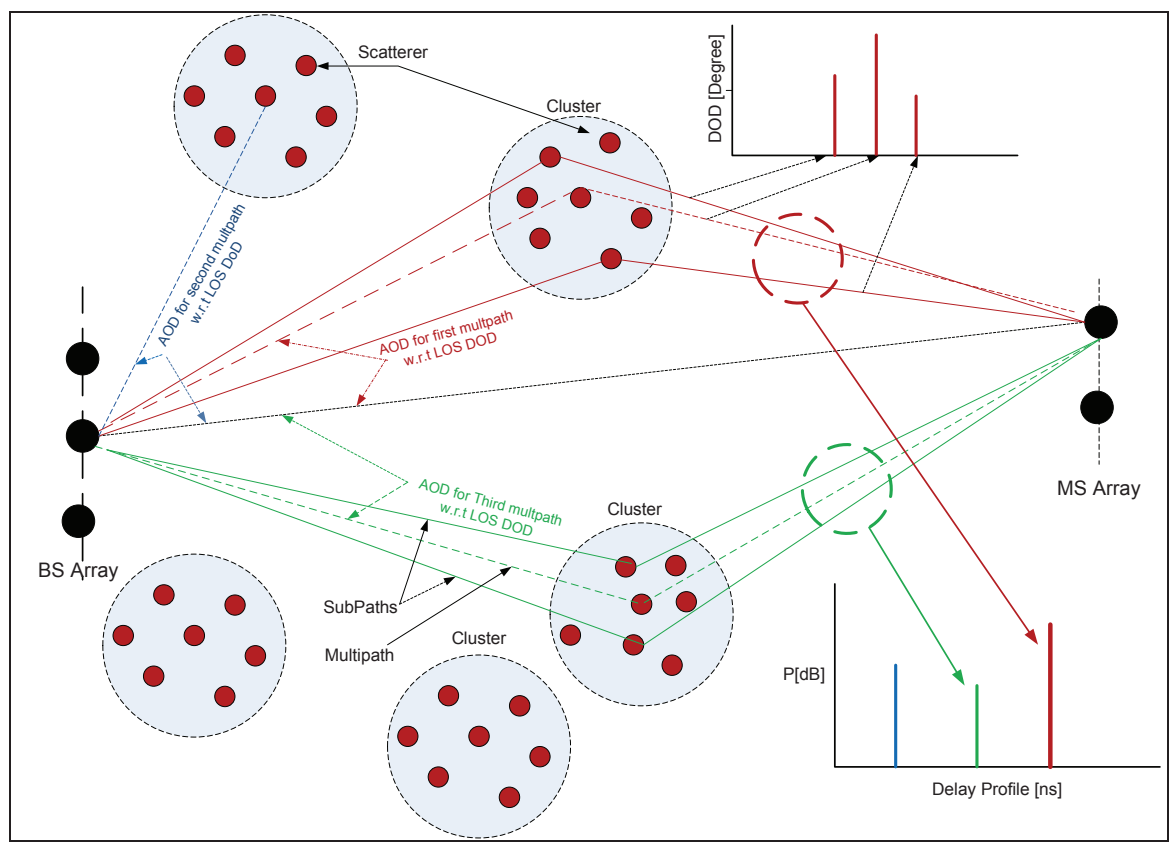

Fig. 5. Clustered scatterers, multipaths and sub-paths in an SCM simulation.

\subsubsection{Software implementation of SCM}

The modelling approach can be divided in to three parts: (i) antenna correlation (ii) spatial correlation and (iii) polarisation correlation. This is illustrated schematically in Fig. 6 which represents the impulse response of the $n^{\text {th }}$ multipath component. 


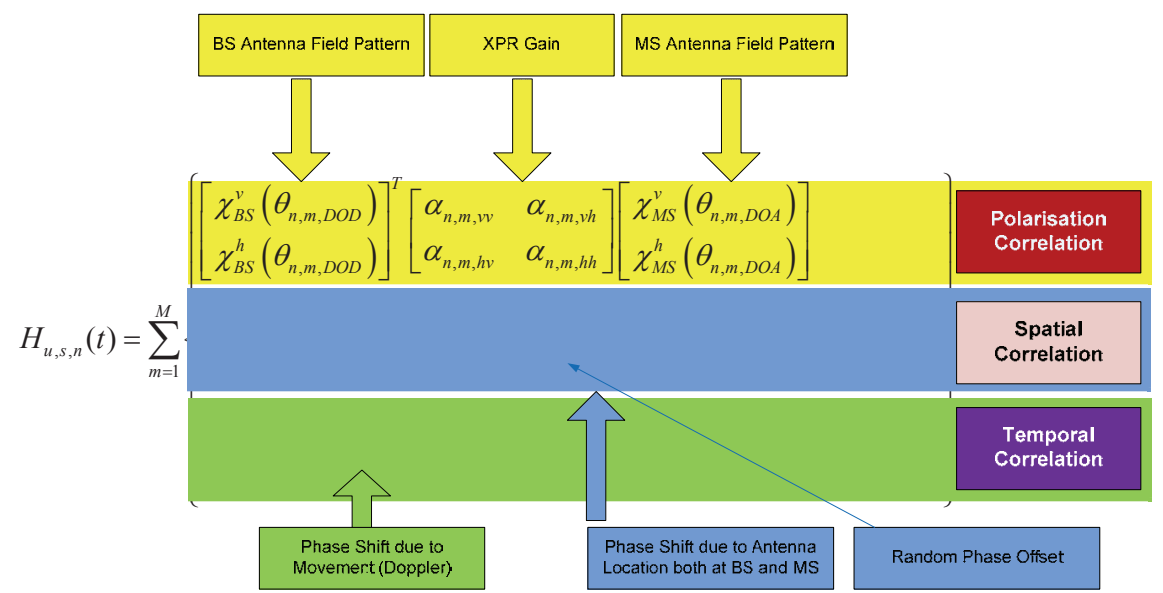

Fig. 6. Tap $n$ complex gain for SCM

Where $H_{u, s, n}(t)$ is the complex gain of the $n^{\text {th }}$ tap (corresponding to the $n^{\text {th }}$ MPC) between the $s^{\text {th }}$ element of a linear BS array and the $u^{\text {th }}$ element a linear MS array. $x^{v} v_{B S}\left(\theta_{n, m, D O D}\right)$ and $x_{B S}^{h}\left(\theta_{n, m, D O D}\right)$ are, respectively, the BS antenna complex field patters for vertically and horizontally polarised fields. Matrix $\alpha_{n, m}$ represents cross-polarised coupling between vertically and horizontally polarised components. $x_{M S}^{v}\left(\theta_{n, m, D O A}\right)$ and $x^{h} M S\left(\theta_{n, m, D O A}\right)$ are, respectively, the MS antenna complex field patterns for vertically and horizontally polarised fields. (Note that the DOD and DOA are each defined by a single angle. This reflects the assumption that there is spreading in azimuth only. Whilst energy is unlikely to be completely constrained to a single azimuthal (horizontal) plane in reality the spread of energy in elevation (out of the horizontal plane) is likely to be relatively modest. The spatial correlation is a function of separation between BS and MS antenna array elements and their respective DODs and DOAs. A random phase offset $\varphi_{n, m}$ (uniformly distributed between 0 and $360^{\circ}$ ) is added to ensure a random starting point for fast fading. Temporal correlation is a function of the magnitude and direction of MS motion. Fig. 7 shows a pseudo-code flow diagram for the model. The simulation steps implied by Fig. 7 are: 


\begin{tabular}{|l|}
\hline \multicolumn{1}{|c|}{ Legend } \\
$\mathrm{L}=$ Number of MIMO links \\
$\mathrm{N}=$ Nurnber of Multipaths \\
$\mathrm{M}=$ Number of Subpaths \\
$\mathrm{T}=$ Number of Fading Samples
\end{tabular}

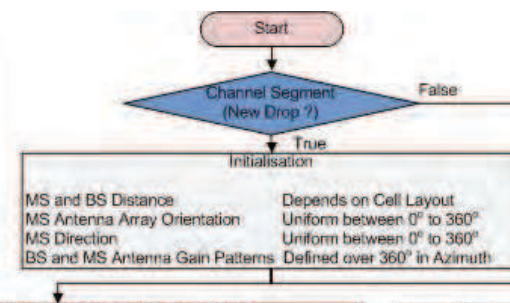

\begin{tabular}{|c|c|}
\hline \multicolumn{2}{|c|}{$\downarrow$} \\
\hline \multicolumn{2}{|c|}{ Determine Channel Parameters (based on user input) } \\
\hline Path Delay & Unilormly Distributed \\
\hline Average Power in each Tap & Gaussian Distributed \\
\hline Direction of Deparure (for each multipa:h) & Gaussian Distributed \\
\hline Clustering (of each multipath) at the BS & $\begin{array}{l}\text { Determine Power. Phase and Offset } \\
\text { for each Subpath in a Cluster. }\end{array}$ \\
\hline Direction of Arrival (for each multipath) & Gaussian Distributed \\
\hline Clustering (of each multipath) at the MS & $\begin{array}{l}\text { Delermine Power. Phase and Offset } \\
\text { for each Subpath in a Cluster. }\end{array}$ \\
\hline Pairing of Multipath and Subpaths. & Random \\
\hline Pathloss and Shadowing & Deterministic Models \\
\hline Random Phase for each Link. & Unilorm Distributed \\
\hline
\end{tabular}

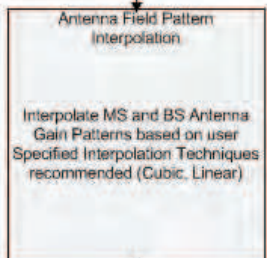

Random Phase Unilorm Distributed

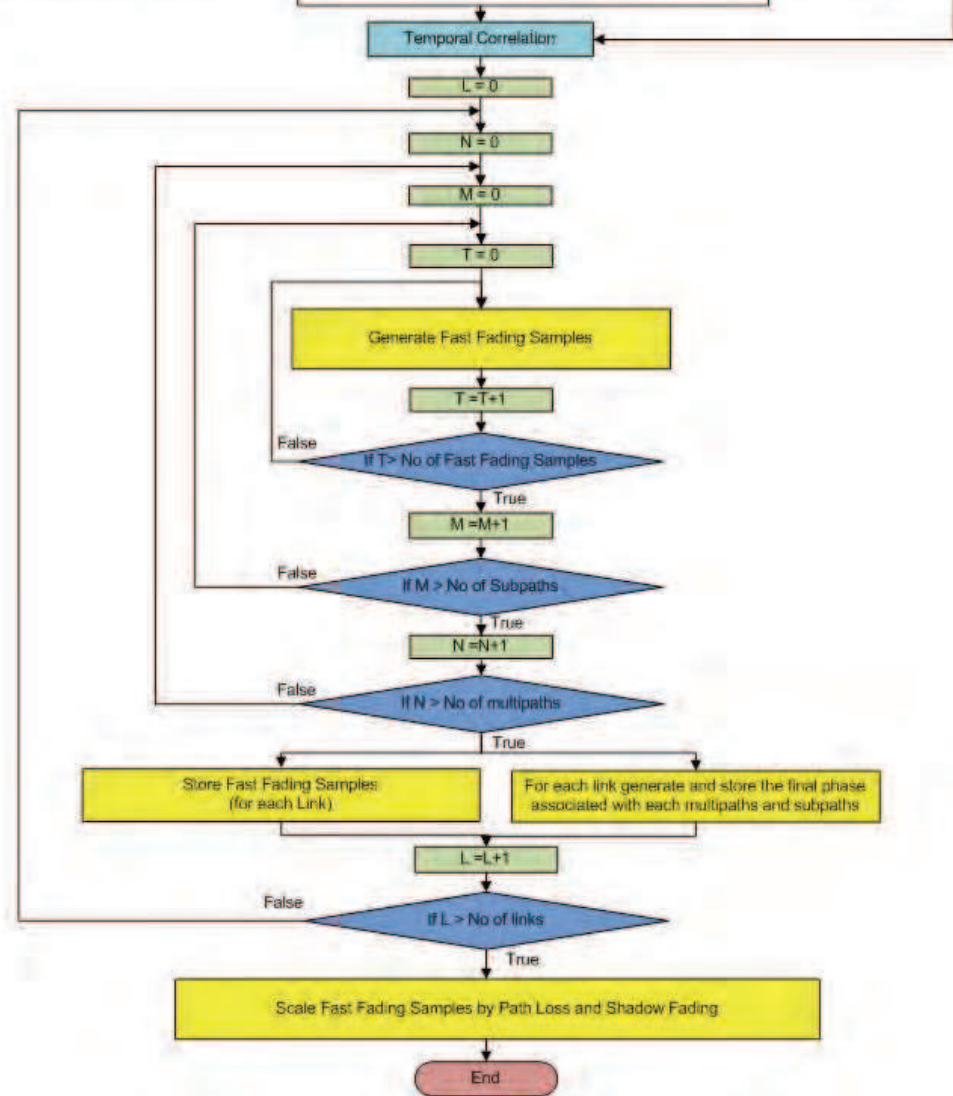

Fig. 7. Pseudo-code for SCM channel model 
1. For a given drop the multiple MSs are placed at random locations within a sector/cell. The MS (linear array) antenna orientation and the MS direction of motion are selected at random.

2. Path-loss is calculated based on the separation between MS and BS. The path-loss model used is the COST 231 (Hata) model for macrocells and the COST 231 (Walfish-Ikegami) model for microcells. The number of multipath components is fixed in all three cases at six and their delay and average power are chosen randomly using appropriate probability distributions.

3. Angular dispersion at the MS and BS is incorporated by assuming each multipath component comprises a cluster of 20 sub-paths having the same delay but (randomly modelled) different DOD and DOA [3GPP TR.996 V6.1.0 (2003 09)]. The overall mean DOD and DOA is determined by the relative locations of BS and MS and the orientation of their antenna arrays. The mean DOD or DOA for each tap is chosen at random from a Gaussian distribution that is centred on the overall mean. (DOA and DOD variances are model parameters.) . The sub-paths have deterministic amplitude and random phase. Their sum is therefore subject to Rayleigh or Ricean fading.

4. Temporal fading may be generated either by using a sum of sinusoids or by applying white Gaussian noise to a Doppler filter.

5. Temporal variation of the impulse response is determined by the speed and direction of the MS. The different Doppler shift for each sub-path leads to a different phase for each sub-path. The phase for each sub-path at the end of each call within a single simulation drop is stored in order to ensure continuity, Fig. 8.

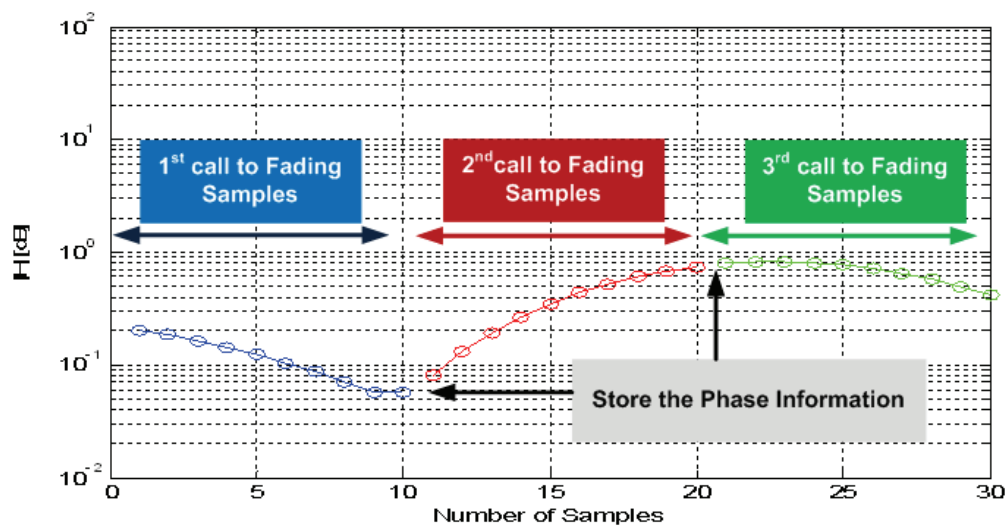

Fig. 8. Fast fading samples for one tap corresponding to successive calls of the same channel drop.

5. For system level simulations (which consider multiple MSs and BSs in different cells/sectors) a sequence of drops is executed. All parameters are independent of those in prior or succeeding drops. The drop period is assumed to be sufficiently short for large-scale channel parameters (e.g. angle spread, mean DOD/DOA, delay-spread and shadowing) to be constant during the drop. The MS position is determined at random during the start of each drop. 


\subsubsection{Model features}

1. Each drop reflects a snapshot of the fading channel.

2. The COST 231 Urban Hata (macrocell) and COST 231 Walfish-Ikegami (microcell) path-loss models are adjusted for a frequency of $1.9 \mathrm{GHz}$. The applicability to a frequency other than $1.9 \mathrm{GHz}$ is not analysed [M. Narandzic., 2007].

3. The model provides a bandwidth of $5 \mathrm{MHz}$ which is not adequate for the most recent wireless standards (e.g. LTE which requires bandwidth of $20 \mathrm{MHz}$ ).

4. During a drop the channel undergoes fast fading due to MS movement. Delays, DOD and DOA, however, are kept constant. Any two consecutive drops are independent and are based on randomly located clusters. This makes the channel model discontinuous across drops.

5. Six clusters of scatterers are considered. Each cluster corresponds to a resolvable MPC (referred to as a multipath). Within a resolvable path (cluster), there are 20 irresolvable sub-paths. Each of the multipaths is modelled as a Dirac (delta) function of delay. Each multipath is subject to angular dispersion across its 20 sub-paths. The summing of the sub-path carriers results in Rayleigh fading of each multipath [D.S. Baum., 2005].

6. The correlation of the standard deviation (in $\mathrm{dB}$ ) of lognormal fading due to shadowing (between the links from a single MS to multiple BSs) is 0.5. The correlation is independent of the range of BSs or their relative angular locations as seen from the MS. Shadowing and its correlation between multiple MS-BS paths are therefore assumed independent of network topology and topography [3GPP TR25.996 V6.1.0, 2003-09].

7. Elevation spread is not considered.

8. Antenna radiation pattern, array geometry and array orientation are arbitrary. When all propagation and antenna parameters are defined an analytic formulation can be extracted from the physical model. Each drop results in a different instance of the correlation matrix.

9. Most model parameters are described by their PDFs. While this provides a rich source of variability, it has the disadvantage that simulation time grows exponentially with the number of random parameters.

10. As a consequence of the assumption that each multipath is flat-faded, the model's utility for bandwidths above $5 \mathrm{MHz}$ is questionable. (This motivates the extended, SCME, version of SCM.)

11. Uplink and downlink reciprocity is assumed, i.e. DOD and DOA values are identical for both uplink and downlink simulation of the channel.

12. The uplink and downlink sub-path (random) phases are uncorrelated for frequency division duplex (FDD) systems but correlated for TDD systems. .

\subsubsection{Extended Spatial Channel Model (SCME)}

SCME is an extension of SCM. The extension is not associated with the 3GPP working group but was developed in WP5 of the WINNER project. SCME extends the channel bandwidth of SCM from $5 \mathrm{MHz}$ to $20 \mathrm{MHz}$. It was adopted as the channel standard for the development and testing of the 3GPP Long Term Evolution (LTE) standard. The channel bandwidth was subsequently further extended to $100 \mathrm{MHz}$. (The required bandwidth of B3G systems is up to $100 \mathrm{MHz}$ in both $2 \mathrm{GHz}$ and $5 \mathrm{GHz}$ bands.) A limitation of SCM is the 
drop-based approach with the consequence that there is no short-term variability in the channel transfer function. This corresponds to fixed DOAs as seen by a moving MS. SCM also has a limited range of scenarios (it does not include, for example, outdoor-to-indoor paths) and, in some scenarios, does not incorporate $K$-factor to support LOS paths.

SCME uses the intra-cluster delay-spread to effect bandwidth extension. Since backward compatibility with SCM was required the number of clusters (i.e. multipaths) is not increased from six. Each cluster of 20 sub-paths (which in SCM have identical delay) is subdivided into 3 or 4 sub-clusters (for macrocell and microcell scenarios respectively) called mid-paths with different delays, Fig. 9.

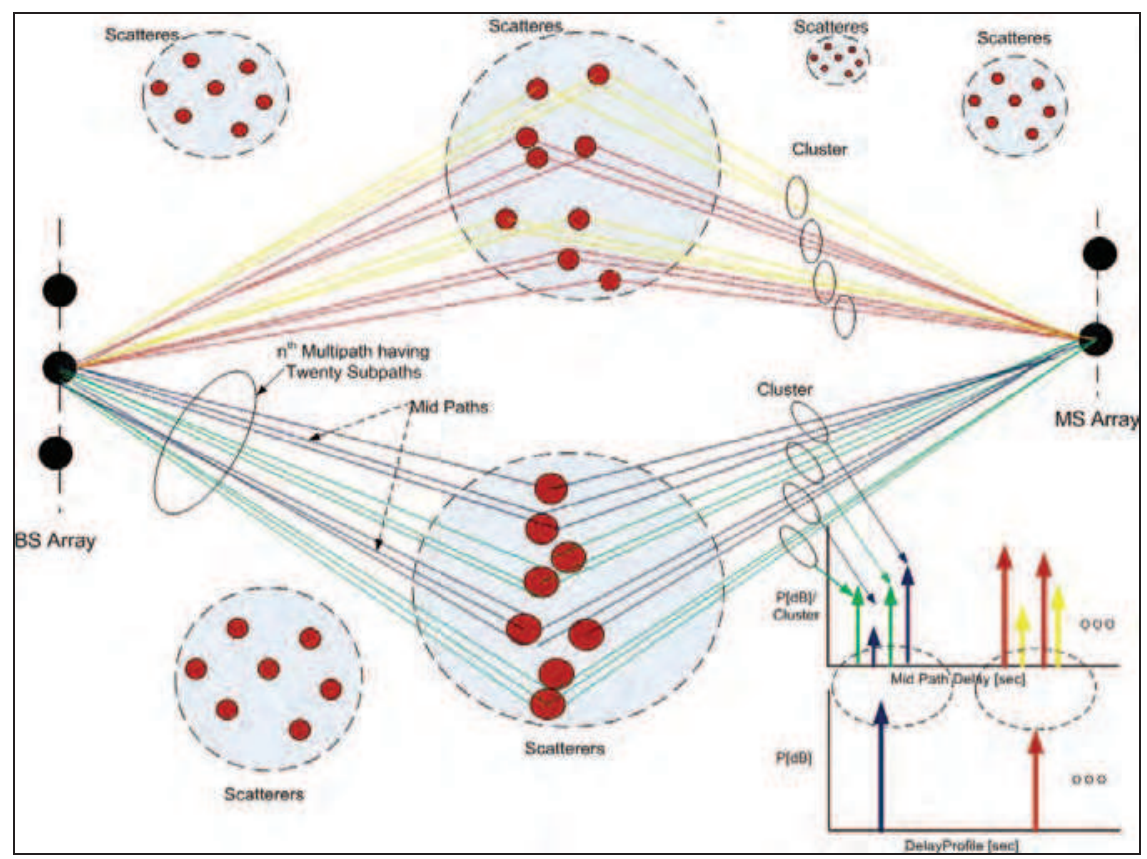

Fig. 9. SCME multipaths, mid-paths and clusters

(The total number of sub-paths for SCME, however, remains the same as for SCM, i.e. 20.) Bandwidth extension is realised by introducing delay (and power) difference between the mid-paths The number of delay taps is therefore increased from 6 in SCM to 18 or 24 in SCME (depending on the scenario [Spirent Communications, 2008]). The 20 sub-paths are split into groups of 10,6, and 4 (for scenarios with three mid-paths) or groups of 6, 6, 4 and 4 (for scenarios with four mid-paths). The relative power of each mid-path is scaled by this ratio as shown in Fig. 10. 


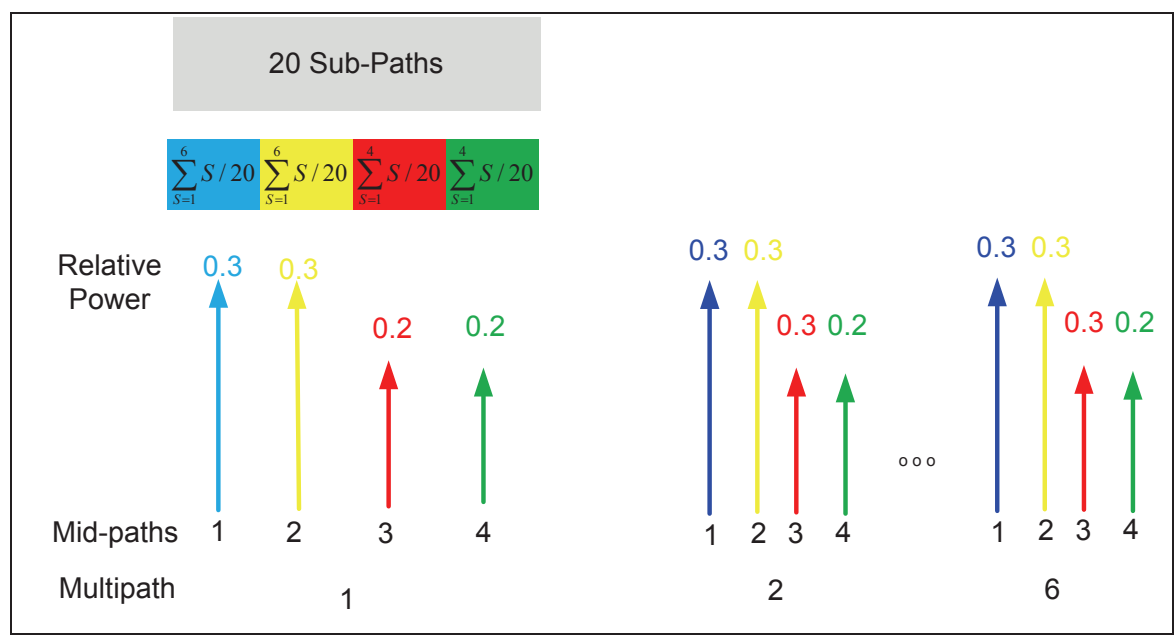

Fig. 10. Multipaths, mid-paths and sub-path in SCME.

Sub-paths within a mid-path have identical delay. Each mid-path has the same azimuth spread as in SCM. SCME is a continuous evolution model since it allows drifting of DODs, DOAs and delays for every multipath at each simulation step within a drop.

\subsubsection{Additional features of SCME}

SCME contains the following additional features when compared to SCM [D.S Baum et. al., 2005; M. Narandzic., 2007; SCME Project., 2005]:

1. Addition of intra-cluster delay-spread within a multipath

The introduction of intra-cluster delay-spread to increase bandwidth. The 20 subpaths of SCM are grouped into three (in case of macrocells) or four (in case of microcells) mid-paths with different delays. This results in about $10 \mathrm{~ns}$ of intra-cluster RMS delay spread. The delays, which are fixed, are given in [D.S Baum et al., 2005]. A mid-path, which represents a single resolvable delay (or tap), comprises a collection of sub-paths and therefore has a fading distribution close to Rayleigh.

\section{Frequency}

Carrier frequency is extended to $5 \mathrm{GHz}$.

\section{Path-loss models}

Two path-loss models are used. The long-range model (identical to that defined in the SCM $2 \mathrm{GHz}$ model) and an alternative, short-range, model. The extension of frequency to $5 \mathrm{GHz}$ is applied to both. SCME selects the path-loss model (long-range/short-range/2 GHz/5 $\mathrm{GHz}$ ) depending on the user input. The $5 \mathrm{GHz}$ path-loss model offsets loss by $+8 \mathrm{~dB}$ with respect to the existing $2 \mathrm{GHz}$ model.

\section{LOS model for all scenarios}


The LOS option affects path-loss and shadow-fading variance. The choice between LOS and NLOS within a drop is based on the probability of LOS versus BS-MS distance. The LOS model in SCM defines a path-loss and Ricean $\mathrm{K}$-factor which is applicable to urban microcell scenario only. With the alternative path-loss model in SCME, the LOS option (with appropriate K-factor model) is defined for all scenarios and is thus available whenever the current drop is LOS.

\section{Time-variant shadowing}

Each drop consists of time samples within a channel snapshot. In SCM the fading due to shadowing is constant for the duration of a drop. In SCME shadow induced fading (in $\mathrm{dB}$ ) changes within a drop thus modelling time-varying shadowing. The decorrelation distance of shadowing is predefined (i.e. $5 \mathrm{~m}, 50 \mathrm{~m}$ and $250 \mathrm{~m}$ in urban microcells, urban macrocells, and suburban macrocells, respectively). The standard deviation of shadow induced fading for all scenarios is $4 \mathrm{~dB}$ for LOS and $10 \mathrm{~dB}$ for NLOS.

\section{Time-variant DODs, DOAs and delays}

For all sub-paths, DODs and distances are calculated once for every channel snapshot. It is assumed, therefore, that the locations of scatterers relative to the BS are fixed for the duration of a drop. This results in fixed DODs as seen by the BS (with the exception of any LOS DOD which does change). The DOAs as seen from the MS and the sub-path delays change during a drop due to the MS movement.

\subsubsection{Model features}

1. SCME is a stochastically controlled spatial channel model. The model is based on the same design philosophy as SCM i.e. the summation of specular components to define the changing impulse response. The model is backward compatible with the existing SCM model.

2. SCME provides a channel bandwidth of up to $100 \mathrm{MHz}$ which is sufficient to characterise B3G wireless technologies and networks.

3. In addition to fast fading (due to multipath propagation), SCME can model the evolution of slow fading (due to shadowing), sub-path delay and DOA during each drop. This results in, time-varying, spatial correlations between transmit and receive antenna elements as shown in Fig. 11. 


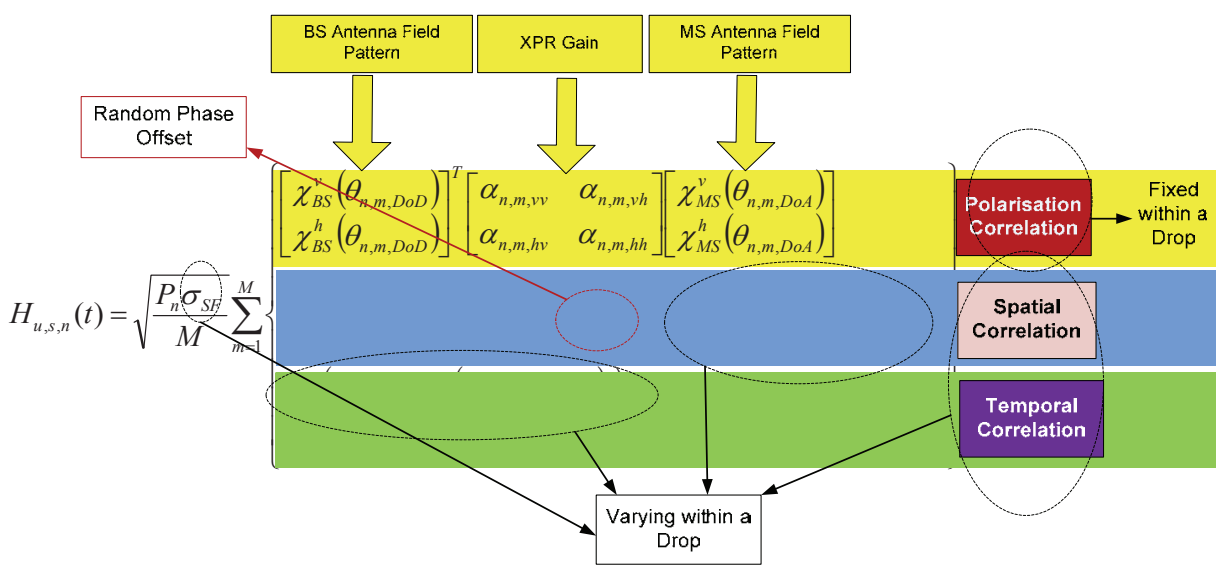

Fig. 11. Tap $n$ complex gain for SCME

4. SCME considers six clusters of scatterers. Each cluster corresponds to a resolvable path. Within a resolvable path (cluster), there are 20 sub-paths. The 20 sub-paths are divided into mid-paths which are then assigned different delays relative to the original path. The mid-paths are delay resolvable but sub-paths within a midpath are irresolvable. The mid-path (which is a collection of subpaths) corresponds to a single tap.

5. SCME includes a LOS K-factor option which is switch selectable for all urban and suburban macrocell scenarios. (SCM includes a LOS model for the urban microcell scenario only.)

6. A simplified tap delay line model referred to as the cluster delay line (CDL) model is used for calibration and comparison purposes. In this model, the DODs and DOAs are fixed for each path. Also, fixed delays are defined resulting in a fixed power delay profile (PDP) [Spirent Communications, 2008].

\subsubsection{Software implementation of SCME}

1. The drop concept in SCM corresponds to a relatively short channel-observation period that is significantly separated from adjacent drops in both time and space. The channel parameters are therefore constant within the drop and independent between drops. Short-term variability of channel parameters within a drop is incorporated by introducing drifting of (i) path delays (ii) DOAs and (iii) shadow induced fading.

2. The position of scatters is fixed within a drop. As a consequence the scattering angles as seen from the BS (DOD) do not change (with the exception of the LOS DOD in LOS scenarios). This assumption is common to SCM. The initial values of random parameters such as DODs, DOAs, K-factor, path phases etc. are generated in the same way as in SCM.

3. The scatter angles as seen from the MS (DOAs) and sub-path delays change (in contrast to SCM) during a drop reflecting MS movement. Similarly the LOS direction from the BS to MS varies in time. This results in time-varying spatial correlation between MS and BS antenna array elements. 
4. An initial value of distance $\left(d_{j, i}\right)$ between the MS and the last bounce scatter (LBS) of the $i^{\text {th }}$ sub-path of the $j^{\text {th }}$ multipath is required in order to calculate DOA drifts as the MS moves. This distance is unknown but can be inferred from a stochastic model as proposed in [D. S. Baum et. al., 2005].

5. Time evolution, or drifting, of slow fading (shadowing) is determined by the spatial auto-correlation function.

Fig. 12 shows the pseudo-code flow diagram for an implementation of SCME.

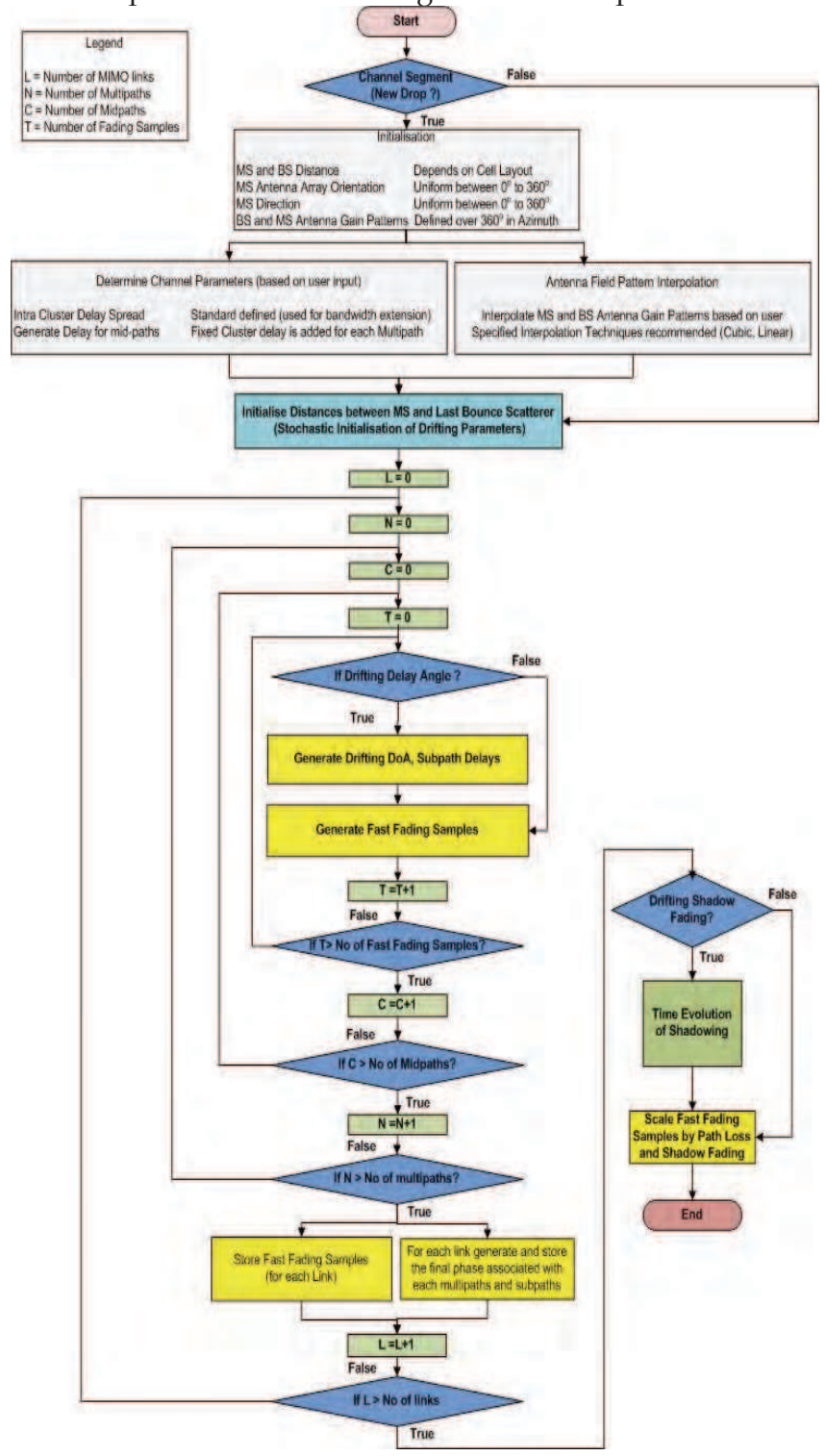

Fig. 12. Pseudo-code flow diagram of SCME channel model. 


\subsubsection{WIM2}

The WIM2 channel model (also referred to as WINNER II [WINNER II Channel Models, 2006]) is defined for both link-level and system-level simulations. It encompasses a wide range of scenarios relevant to local, metropolitan and wide-area systems. WIM2 evolved from the WINNER I and WINNER II (interim) channel models.

WIM2 is a double-directional geometry-based stochastic channel model. It incorporates generic multilink models for system-level simulations and clustered delay line (CDL) models, with fixed large-scale channel parameters, for calibration and comparison purposes.

Initially, the WINNER group selected SCM for immediate simulation but later extended this to SCME. In spite of the greater bandwidth and higher frequency capability of SCME it was still deemed inadequate for advanced WINNER II simulations. The novel features of WIM2 are its parameterisation, the addition of further outdoor and indoor scenarios and the consideration of both azimuth and elevation spreading for indoor environments. (This is in contrast to SCM and SCME which restrict spreading to the azimuthal plane only.) WIM2 also includes correlation modelling of large-scale parameters and scenario-dependent polarisation modelling [WINNER II Channel Models, 2007].

\subsubsection{Model features}

1. It is a geometry-based stochastic channel model, which allows the creation of an arbitrary double-directional model. The channel impulse response is the sum of specular components. This is the same principle as used in the SCM and SCME channel model. The channel parameters are determined randomly, based on probability distributions extracted from channel measurement.

2. It covers 12 scenarios which is a larger number than SCM or SCME. These include indoor environments. (SCM and SCME include only outdoor environment.) Each scenario allows LOS or NLOS conditions. The model supports mobile, nomadic and fixed systems.

3. It allows transitions between different propagation conditions, the most important of which are transitions between LOS and NLOS within the same scenario. In the A1 (indoor) or B1 (urban microcell) scenarios, for example, transitions from LOS to NLOS can occur as a result of the MS turning a corner to leave a corridor or street in which the BS is located.

4. It can be used to characterise systems operating in the $2-6 \mathrm{GHz}$ frequency range with a bandwidth up to $100 \mathrm{MHz}$. Like SCME, intra-cluster delay spread is used to support this bandwidth.

5. It specifies DOA and DOD as two-dimensional variables (whereas only a single (azimuthal) angle is considered in SCM and SCME).

6. It employs a sophisticated correlation model to the multiple links in system level simulations [M. Narandzic et. al., 2007]. (This contrasts with SCM which adopts a fixed correlation (0.5) for slow fading to model multiple links between a MS and different BSs.)

7. A drop, or channel segment, represents a quasi-stationary period during which the probability distributions of most channel parameters do not change. The advantage of this approach is simplicity. The disadvantage is that it is not 
possible to adequately simulate cases where variable channel conditions are needed. Drop-based simulation is the principal approach used by both WINNER I (SCM and SCME) and WIM2 models. WIM2, however, also allows simulation with time evolution in which the drops are correlated and a smooth transition between consecutive drops is engineered. (The drops in WIM2 are usually referred to as channel segments since they are no longer 'dropped in' at random.) This smooth transition between channel segments is realised by spacing the segments in time by the quasi-stationary duration and dividing the transition region into a number of sub-intervals. The number of sub-intervals is chosen to be the same as the higher of the number of clusters in either channel segment. In each sub-interval the strength (amplitude) of a cluster from the earlier segment is ramped linearly down and the strength of the cluster from the later segment is ramped up. The pair of clusters is chosen to be, as far as possible, 'matched' in strength. Where the number of clusters in earlier and later segments are not equal then the weakest paths in the segment with more clusters are left unpaired and are ramped up or down alone. This process is illustrated schematically in Fig. 13.

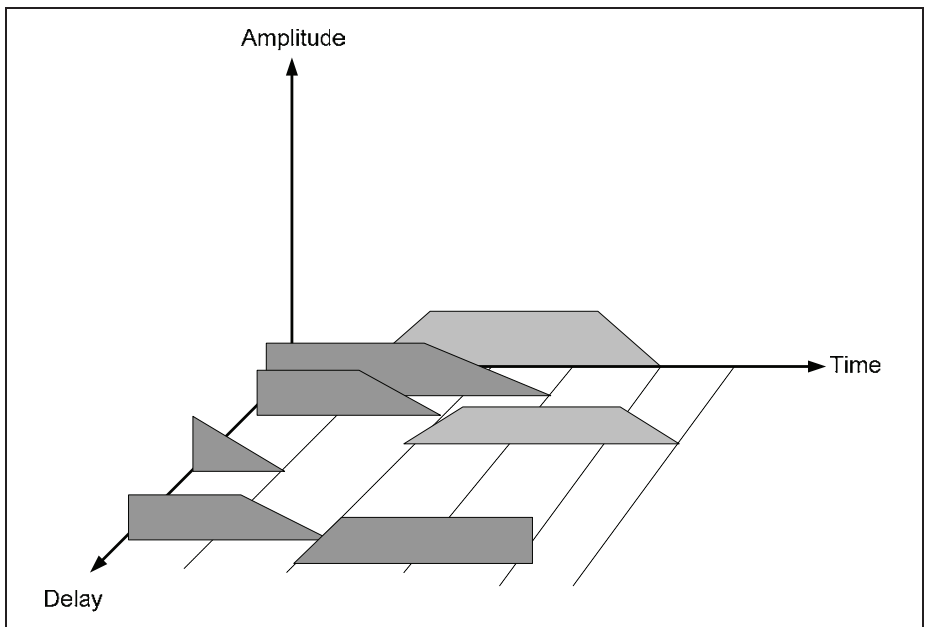

Fig. 13. Transition between channel segments by power ramping up and down of clusters (After [WINNER II Channel Models, 2007]).

8. For time division duplex (TDD) systems WIM2 (like SCM and SCME) uses the same parameters for both uplink and downlink. For frequency division duplex (FDD) systems a different path-loss is used on uplink and downlink and the random phases of sub-paths on uplink and downlink are modelled independently [M. Narandzic et. al., 2007].

\subsubsection{Channel modelling approach}

The modelling process can be divided into three phases. These are illustrated in Fig. 14. 


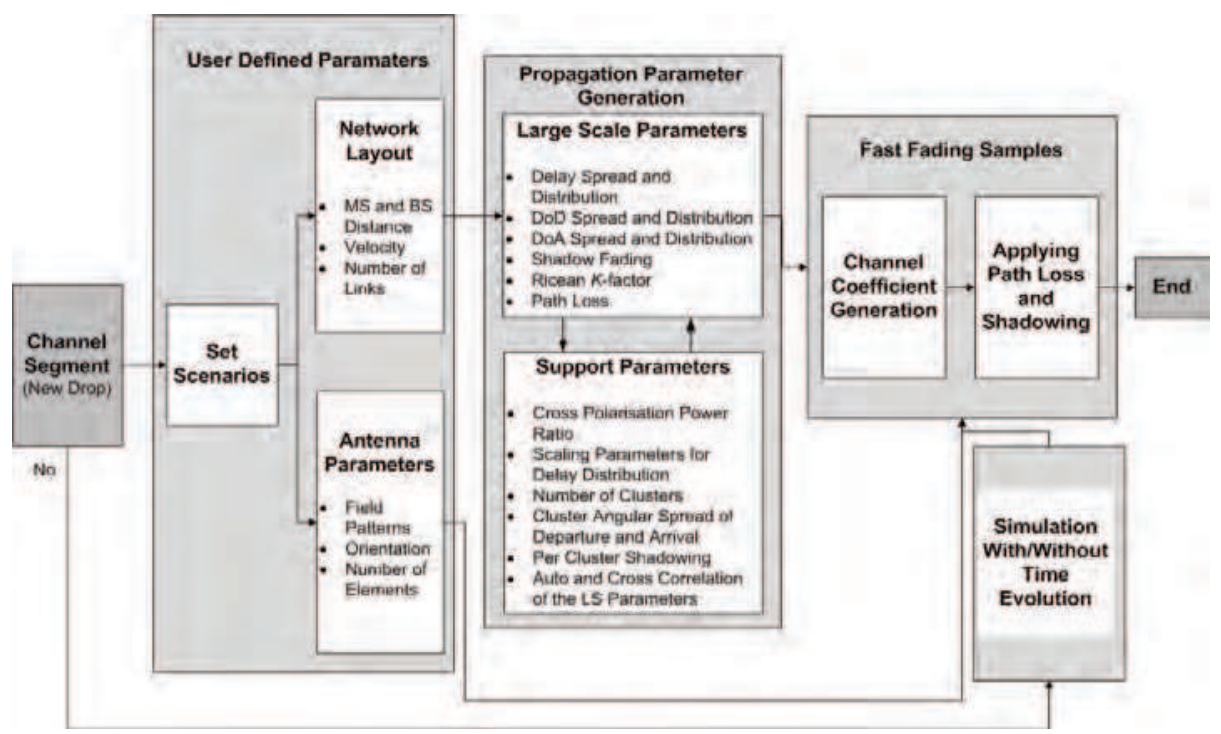

Fig. 14. WIM2 channel modelling process.

1. The environment, network layout and antenna array parameters are defined.

2. The large-scale parameters such as slow fading (shadowing), power, DODs, DOAs and delay-spread are drawn randomly from tabulated distribution functions. At this stage the geometry (i.e. network layout) is fixed and the only free variables are the random initial phases of the sub-paths. By picking different initial phases (randomly), an unlimited number of different realisations of the model can be generated. When the initial phases are fixed, the model is deterministic.

3. Fast fading samples are generated using the sum of sinusoids technique. (This is the same as in SCM and SCME.) The model implements time evolution (depending on user input) in order to generate correlated channel samples if the required channel simulation period is longer than the quasi-stationery period of the channel.

\subsection{WiMAX}

IEEE working group 802.16 has been central to the development of technical standards for fixed wireless access networks. Broadband wireless access (BWA) technology provides last mile access for high-speed residential and commercial Internet services. It is a promising alternative to digital subscriber line (DSL), cable and fibre technologies which are struggling to meet world-wide demand, especially outside metropolitan centres, for Internet services at reasonable cost. The IEEE 802.16 standard for BWA and its associated industry consortium, the WiMAX forum, has the potential to offer broadband access to virtually all users irrespective of location. WiMAX (the Worldwide Interoperability for Microwave Access) is a consortium of telecommunication equipment manufacturers, vendors and service providers, 
formed to promote the compatibility and interoperability of BWA devices incorporating the IEEE 802.16 and ETSI HiperMAN wireless standards.

IEEE 802.16 was designed for LOS links operating at carrier frequencies between 10 and 66 GHz. The first release of the standard (IEEE 802.16-2001) specifies a set of medium access control (MAC) and physical-layer standards intended to provide fixed broadband access using a point-to-point (PP) or point-to-multipoint (PMP) topology. The standard was revised in January 2003 to included NLOS links operating at frequencies in both licensed and unlicensed bands between 2 and 11 GHz. A consolidated standard, IEEE 802.16-2004, was issued in 2004.

IEEE 802.16e-2005, was issued in December 2005 which includes enhancements for physical and MAC layers that support nomadic and mobile operation in 2 to $11 \mathrm{GHz}$ range.

The WiMAX forum has adopted the IEEE 802.16-2004 and ETSI HyperMAN standards for fixed and nomadic access and the IEEE 802.16e standard for portable access.

Two channel models are used for fixed and portable systems complying with the IEEE 802.16 standard. The Stanford University Interim (SUI) channel model is used for fixed broadband access and the ITU Tapped-Delay-Line channel model is used for portable broadband access.

\subsubsection{SUI}

The Stanford University Interim (SUI) suite of channel models was designed for fixed macrocell networks operating at $2.5 \mathrm{GHz}$. It contains the definition of six specific channel implementations which were initially developed for Multipoint Microwave Distribution Systems (MMDSs) in the USA operating in the $2.5-2.7 \mathrm{GHz}$ frequency band. Their applicability to the $3.5 \mathrm{GHz}$ frequency band that is in use in the UK has so far not been conclusively established.

The model generates both SISO and MIMO channel parameters. The six specific channel implementations represent different terrain types. The targeted scenarios are based on the following assumptions:

1. Cell radius less than $10 \mathrm{~km}$.

2. Cell coverage (80 - $90 \%)$.

3. Fixed directional receiving antenna installed at a height of $2-10 \mathrm{~m}$ (below rooftop height since LOS is not required).

5. Base-station antenna installed at a height of $15-40 \mathrm{~m}$ (above rooftop height).

6. $\quad$ Flexible channel bandwidth between 2 and $20 \mathrm{MHz}$.

Three terrain types are defined: A for hilly terrain with moderate to heavy tree density (representing the highest path-loss terrain type), B for either flat terrain with moderate to heavy tree density or hilly terrain with light tree density and $\mathrm{C}$ for flat terrain with moderate to light tree density (representing the lowest path-loss terrain). 
The SUI models corresponding to these terrain types are:

1. A: SUI-5 and SUI-6

2. B: SUI-3 and SUI-4

3. C: SUI-1 and SUI-2

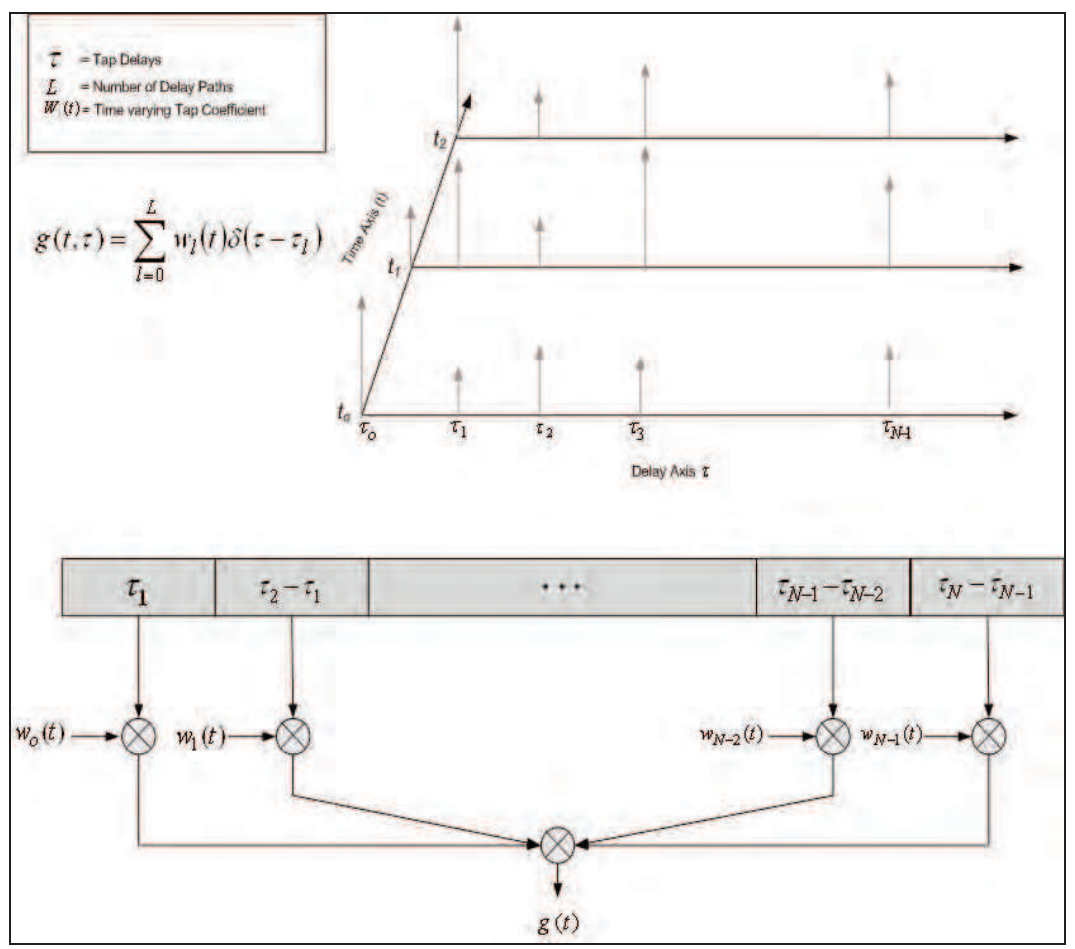

Fig. 15. Tapped delay line channel model. (After[T. S. Rapport, 2002])

\subsubsection{Model features}

1. Claimed to be valid between 2 and $4 \mathrm{GHz}$ (although no tests of its performance in the European $3.5 \mathrm{GHz}$ band are known) and for up to $7 \mathrm{~km}$ separation between transmitter and receiver.

2. Fading modelled as tapped delay line, Fig. 15, with three taps having nonuniform delays. The delays are specified in the standard document [IEEE 802.16 (BWA), 2003].

3. Omni-directional antennas are assumed at both transmitter and the receiver in the original SUI models. A modified version of the model assumes directional antennas with $30^{\circ}$ beamwidths.

4. A lognormal model [L.J. Greenstein et. Al., 1999] for the distribution of $K$-factor is adopted. The $K$-factor for each tap is specified by exceedance values corresponding to cell coverage areas of $90 \%$ and $75 \%$ (SUI-1 to SUI-4) and $90 \%$, 
75\% and 50\% (SUI-5 to SUI-6). Taps 2 and 3 are always, effectively, Rayleigh faded. Tap 1 may be Ricean or Rayleigh faded.

5. Correlation between multipath components (tap weights) for the same taps of different receiving antennas at the MS is fixed irrespective of MS array configuration. The correlation between multipath components for the same taps of different receiving antennas at the BS is zero (corresponding to an assumption of large antenna spacing.

6. Channel coefficients with the probability distribution and power spectral density specified in the standard document [IEEE 802.16 (BWA), 2003] are generated by filtering noise. In a fixed wireless system the Doppler power spectral density is concentrated around $f=0 \mathrm{~Hz}$. The shape of the spectrum, which different from the classical Jakes spectrum (that is more relevant to urban mobile scenarios), is given by:

$$
S\left(f_{D}\right)=\left\{\begin{array}{cc}
1.72\left(f_{D} / f_{m}\right)^{2}+0.785\left(f_{D} / f_{m}\right)^{4} & f_{D} \leq f_{m} \\
0 & f_{D}>f_{m}
\end{array}\right\}
$$

where $f_{m}$ is the maximum Doppler frequency. This is usually referred to as the 'rounded' Doppler spectrum [IEEE 802.16 (BWA), 2003].

7. Validity restricted to distances less than $7 \mathrm{~km}$ (which is less than the targeted maximum range of $10 \mathrm{~km}$ ). A modified version of SUI channel model has been proposed in order to scale transmit-receive distances to ranges greater than $7 \mathrm{~km}$.

8. Propagation environments characterised in terms of power delay profiles.

9. Actual antenna array configurations not considered.

10. Simple and suited for rapid proto-typing or equipment/algorithm development. Unsuited to comprehensive, location-specific, network/system planning.

\subsubsection{Generic structure}

The generic structure of the SUI channel model is shown in Fig. 16 [IEEE 802.16 (BWA), 2003].

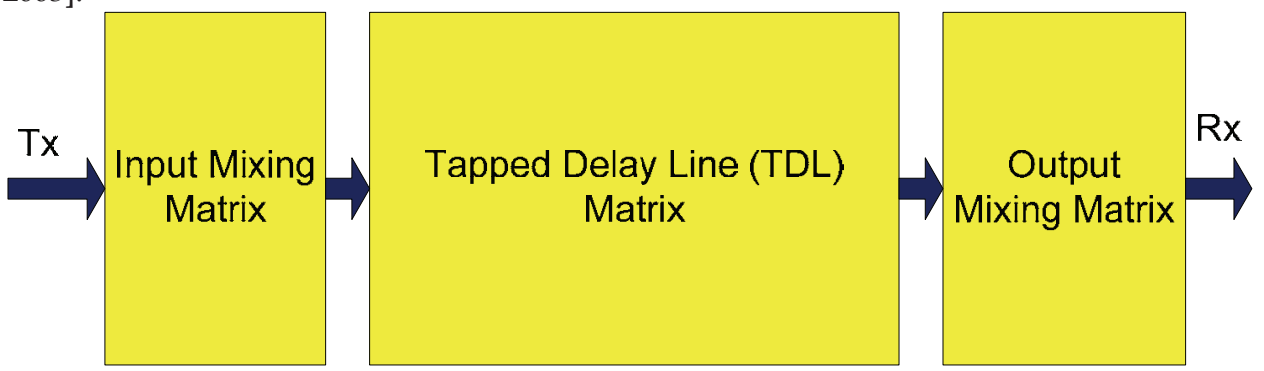

Fig. 16. Generic structure of SUI channel model. (After [IEEE 802.16 (BWA), 2003].)

The input mixing matrix models the spatial correlation between inputs if multiple antennas are used at the transmitter. The tapped delay line matrix models multipath fading. Each filter tap is characterised by a Ricean or Raleigh fading process. The output mixing matrix models the spatial correlation between output signals if multiple antennas are used at the receiver. 


\subsubsection{Channel modelling approach}

1. RMS delay-spread is calculated based on user specified parameters.

2. A set of complex zero-mean Gaussian random numbers are generated for each tap leading to a Rayleigh distributed tap magnitude. If a Ricean distribution $(K>$ 0 ) is required a constant path component is added to the Rayleigh set of coefficients.

3. The random numbers generated are independent (and therefore uncorrelated) and thus have a white spectrum. The SUI channel model specifies a 'rounded' power spectrum. The uncorrelated samples are filtered to generate channel coefficients with the required correlation properties.

4. An antenna envelope correlation value (i.e. the correlation between the amplitude of signals received at corresponding taps of two antenna elements) is defined for multiple transmit or receive elements.

The simulation steps are summarised in Fig. 17.

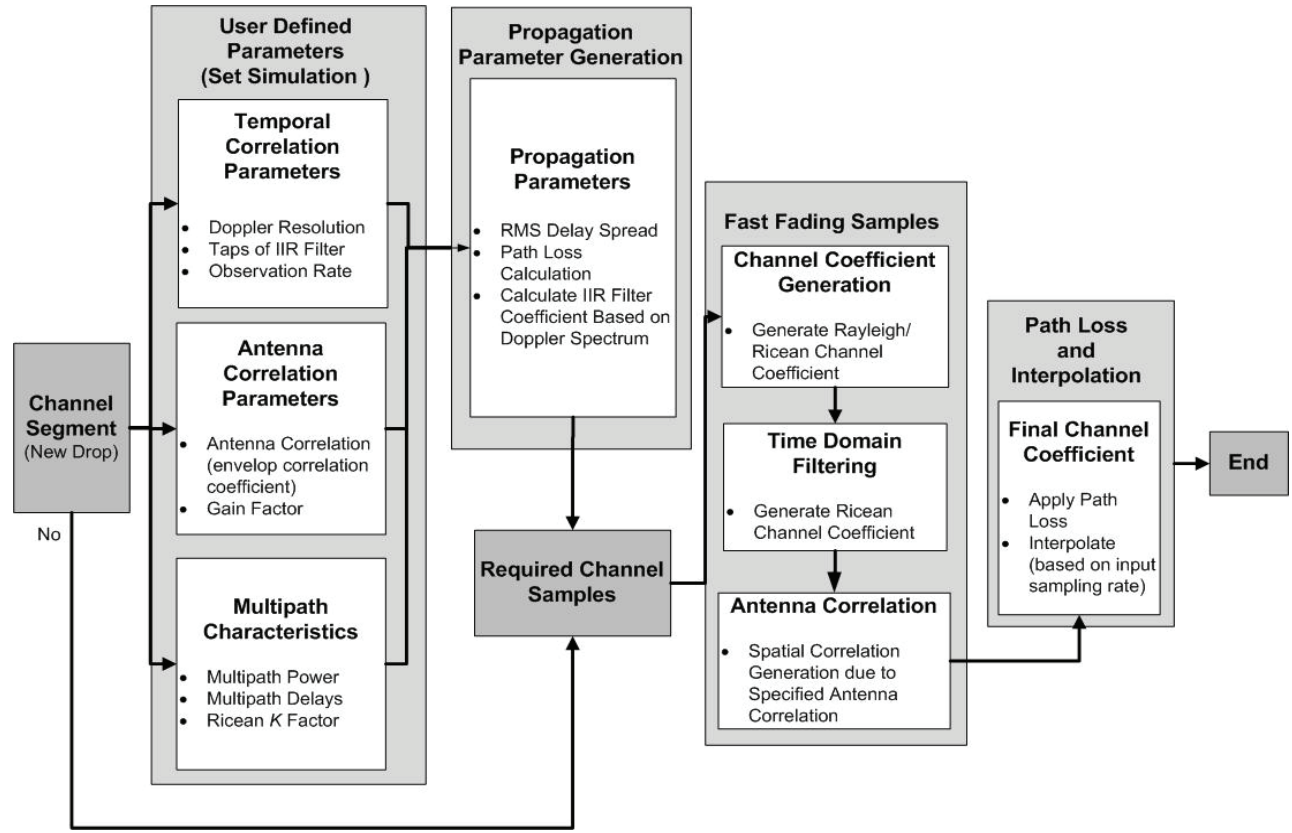

Fig. 17. SUI channel modelling process

\subsubsection{WiMAX- ITU-TDL}

The WiMAX forum approved the mobile WiMAX system profile in 2006. Mobile WiMAX, based on 802.16e-2005, enables WiMAX systems to address portable and mobile devices in addition to fixed and nomadic applications. The WiMAX forum Mobile release 1.0 channel model [WiMAX forum, 2008] defines the SISO and MIMO channel model requirements for mobile applications governed by the IEEE 802.11e standard. The purpose of the model is to provide a realistic and repeatable channel context for the testing and comparison of portable and mobile WiMAX-enabled devices. 


\subsubsection{Model features}

Mobile WiMAX specifies SISO/MIMO channel models for radio conformance testing (RCT) of WiMAX products. The WiMAX forum has selected the ITU Pedestrian-B and Vehicular-A 6-tap TDL models for test and verification of SISO devices. The WiMAX-ITU-TDL channel model extends the ITU-TDL model to the MIMO systems encompassing spatial correlation for each multipath component. One of the novel features of the model is the definition of three levels of channel correlation (low, medium and high) between antenna elements. The following are the main features of the model:

1. It is a physical model, using a stochastic modelling approach similar to SCM.

2. ITU propagation scenarios are extended by defining the azimuth angle spread and shape (Laplacian) of the azimuth spectrum for each tap. Tap-wise MIMO correlation matrices are determined based on the spatial information (i.e. high, medium and low antenna correlation) of multipath components combined with specific antenna configurations.

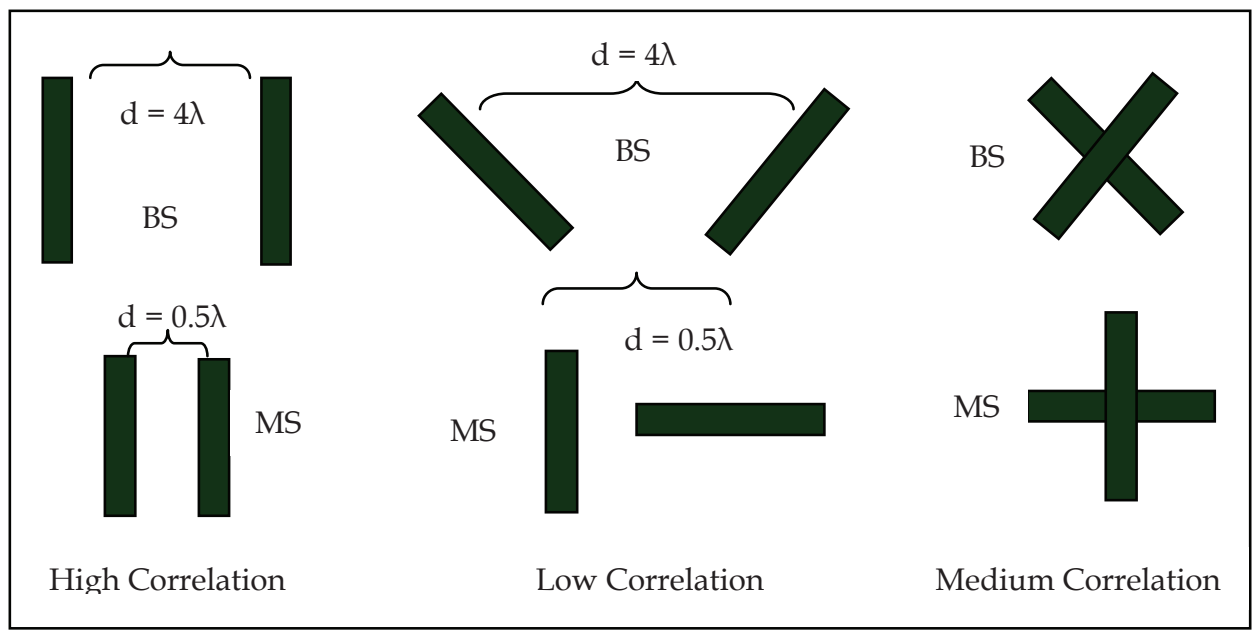

Fig. 18. Three levels of MIMO correlation for WiMAX-ITU-TDL. (After [WiMAX forum, 2008].)

3. Three pairs of reference antenna configurations are defined that typify low, medium and high correlation. The reference configurations specify the spacing of elements and their relative polarisations at MS and BS. This is illustrated schematically in Fig. 18.

4. The azimuth spread in all WiMAX-ITU-TDL scenarios for all taps is $2^{\circ}$ at the BS and $35^{\circ}$ at the MS.

5. The Doppler spectrum has the classical Jakes U-shape [Jakes model].

6. The strength of each tap is Raleigh distributed.

7. To model long channel impulse response $(>10 \mu \mathrm{s})$ and for high-speed MSs $(>120$ $\mathrm{km} / \mathrm{h}$ ), the ITU Vehicular A channel is used but modified such that the last tap is 
moved from $2510 \mathrm{~ns}$ to $10,000 \mathrm{~ns}$. (The strength of the last tap remains unchanged at $-20 \mathrm{~dB}$.)

8. Each cluster corresponds to a resolvable multipath. Within each of the six multipaths there are 20 irresolvable sub-paths. Each of the six multipaths is modelled as Dirac (delta) function of delay. The 20, spatially separated, sub-paths have equal delay.

\subsubsection{WiMAX-ITU-TDL model design}

1. The model can be separated into three components addressing (i) spatial correlation, (ii) polarisation correlation and (iii) temporal correlation. The spatial and polarisation correlation are modelled using square matrices of dimensions $(K \times M \times N) \times(K \times M \times N)$ where $K$ is the number of multipaths, and $M$ and $N$ are the number of elements in the transmit and receive antenna arrays respectively. The temporal correlation matrix has same number of rows as the spatial or polarisation matrices and the same number of columns as the number of required channel samples.

2. Spatial correlation is calculated independently at transmit and receive antenna and is based on antenna geometry. A $2 \times 2$ MIMO system results in the following spatial correlation matrices at transmit and receive antenna elements:

$$
\begin{aligned}
\mathrm{R}_{\mathrm{BS}} & =\left[\begin{array}{ll}
1 & \alpha \\
\alpha & 1
\end{array}\right] \\
\mathrm{R}_{\mathrm{MS}} & =\left[\begin{array}{ll}
1 & \beta \\
\beta^{*} & 1
\end{array}\right]
\end{aligned}
$$

where $a$ and $\beta$ are the spatial correlations between antenna elements at BS and MS respectively. The MIMO correlation matrix is given by the Kronecker product of independent spatial correlation matrices, i.e.:

$$
\mathbf{R}_{\mathrm{S}-M I M O}=\mathbf{R}_{\mathrm{BS}} \otimes \mathbf{R}_{\mathrm{MS}}=\left[\begin{array}{cc}
\mathrm{R}_{M S} & \alpha \mathbf{R}_{M S} \\
\alpha^{*} \mathrm{R}_{M S} & \mathrm{R}_{M S}
\end{array}\right]=\left[\begin{array}{cccc}
1 & \beta & \alpha & \alpha \beta \\
\beta^{*} & 1 & \alpha \beta^{*} & \alpha \\
\alpha^{*} & \alpha^{*} \beta & 1 & \beta \\
\alpha^{*} \beta^{*} & \alpha^{*} & \beta^{*} & 1
\end{array}\right]
$$

3. The use of the Kronecker product corresponds to an assumption that the correlation between elements in the received antenna array is not affected by changes in the spatial configuration of elements in the transmit antenna array. 
4. The polarisation model (which originated in the ITU) assumes a cross-polar ratio $(\mathrm{XPR}=$ cross-polar power $/$ co-polar power $)$ of $-8 \mathrm{~dB}$ for each tap [WiMAX forum, 2008]. The polarisation matrix is denoted by:

$$
\mathbf{S}=\left[\begin{array}{ll}
\boldsymbol{S}_{v v} & \boldsymbol{S}_{v h} \\
\boldsymbol{S}_{h v} & \boldsymbol{S}_{h h}
\end{array}\right]
$$

where $v$ and $h$ denote vertical and horizontal polarisation respectively. (The first subscript refers to the BS and the second subscript refers to the MS.) The downlink polarisation correlation matrix for a $2 \times 2$ MIMO system [WiMAX forum, 2008] is given by:

$$
\mathbf{R}_{\mathbf{P}, \text { MIMO }}=\left[\begin{array}{cccc}
1 & \gamma & 0 & 0 \\
\gamma & 1 & 0 & 0 \\
0 & 0 & 1 & -\gamma \\
0 & 0 & -\gamma & 1
\end{array}\right]
$$

where $\gamma$ depends on XPR (expressed as a power ratio). The structure of $\mathbf{R}_{\mathbf{P}, \mathbf{M I M O}}$ depends on the MS and BS antenna polarisations and orientations. Further detail can be found in [WiMAX forum, 2008].

5. Temporal correlation refers to the auto-correlation of the signal received at a single antenna element. There are two ways of generating temporal (fast fading) tap coefficients. Since the Doppler spectrum is classical and the amplitude is Rayleigh distributed the temporal fading samples can be generated using either the sum of sinusoids technique (Jakes' method) or by filtering white Gaussian noise. Fig. 19 summarises the process of generation of channel weights. 


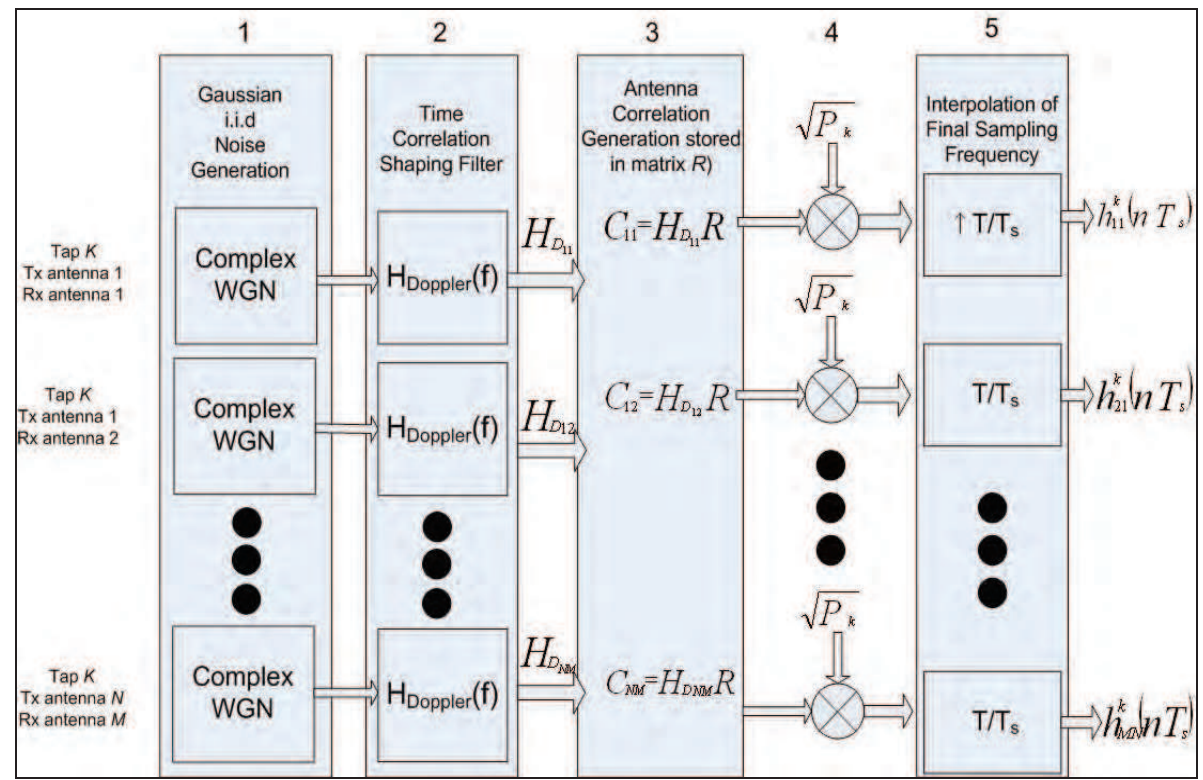

Fig. 19. Block diagram of correlated channel tap generation. (After [WiMAX forum, 2008].)

\subsubsection{Software implementation of WiMAX-ITU-TDL}

Fig. 20 shows pseudo-code for a WiMAX-ITU-TDL channel model. The modelling process is divided into the following principal components: 


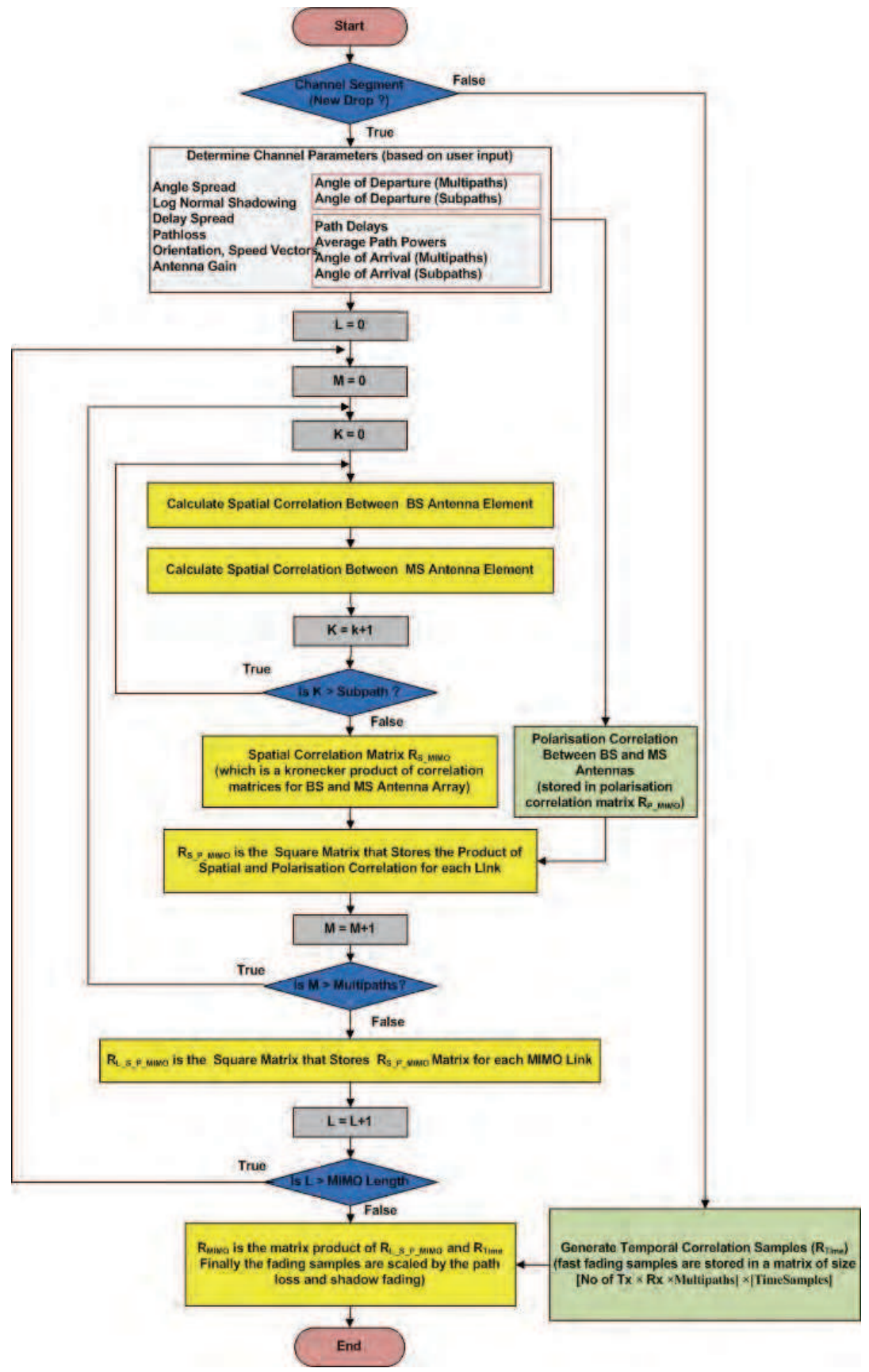

Fig. 20. Pseudo-code flow diagram for WiMAX-ITU-TDL channel model. 
The generation of secondary channel statistics (e.g azimuth spread, offset DOA and offset DOD) is based on the user specified scenario. The channel segment (drop) represents a quasi-stationary period in which the probability distributions of parameters are unchanged. Unlike SCME the underlying large-scale parameters such as DOD and DOA remain constant within a single a channel segment.

The spatial correlation matrices for the BS and MS antenna elements are generated for all multipaths of each link. The BS and MS antenna spatial correlation matrices are Kronecker multiplied. The spatial correlation matrix remains unchanged between multiple drops of the same simulation.

Like spatial correlation the polarisation matrix remains unchanged between multiple drops of the same simulation.

Temporal correlation refers to the autocorrelation of the signal received at a single antenna element. During each drop of the simulation fast fading samples of the tap weights are generated either by the summing sinusoids or filtering a white Gaussian random process.

\subsection{IEEE 802.11n}

IEEE 802.11n is an amendment to the IEEE 802.11-2007 wireless local area network (WLAN) standard [IEEE 802.11, 2009]. It gives higher network throughput compared with earlier variants of the standard, e.g. $802.11 \mathrm{~b}$ and $802.11 \mathrm{~g}$. The maximum raw (physical-layer) data rate is $600 \mathrm{Mbit} / \mathrm{s}$. The IEEE 802.11n channel model [V. Erceg et. al., 2004] was developed for systems using the $2 \mathrm{GHz}$ and $5 \mathrm{GHz}$ bands operating in indoor environments and employing MIMO technology. Measurement results from these two frequencies bands were combined to develop the model. Only the path-loss element of the model depends on frequency. The channel model is based on the clustering approach developed by Saleh and Valenzula [A. A. M. Saleh et. al., 1987] and illustrated in Fig. 21.

\subsubsection{Model features}

The principal features of the model [P. Almer et. al., 2007] are:

1. It is a physical model based on stochastic modelling approach.

2. It can be applied to both $2 \mathrm{GHz}$ and $5 \mathrm{GHz}$ frequency bands.

3. A dual-slope, frequency dependent, model for path-loss is adopted.

4. The channel impulse response is a sum of clusters where each cluster consists of up to 18 multipaths. (The precise number depends on the scenario.) These multipaths are modelled as filter taps which are separated in delay by at least $10 \mathrm{~ns}$.

5. Five scenarios are defined [P. Almer et. al., 2007]: A, B, C, D, E and F. Scenarios A-C represent small environments (RMS delay-spread $<30 \mathrm{~ns}$ ), such as residential homes and small offices. (Scenario $A$ is optional and not recommended for system performance comparison.) Scenarios D-F represent large open spaces with maximum RMS delay-spread <150 ns. 


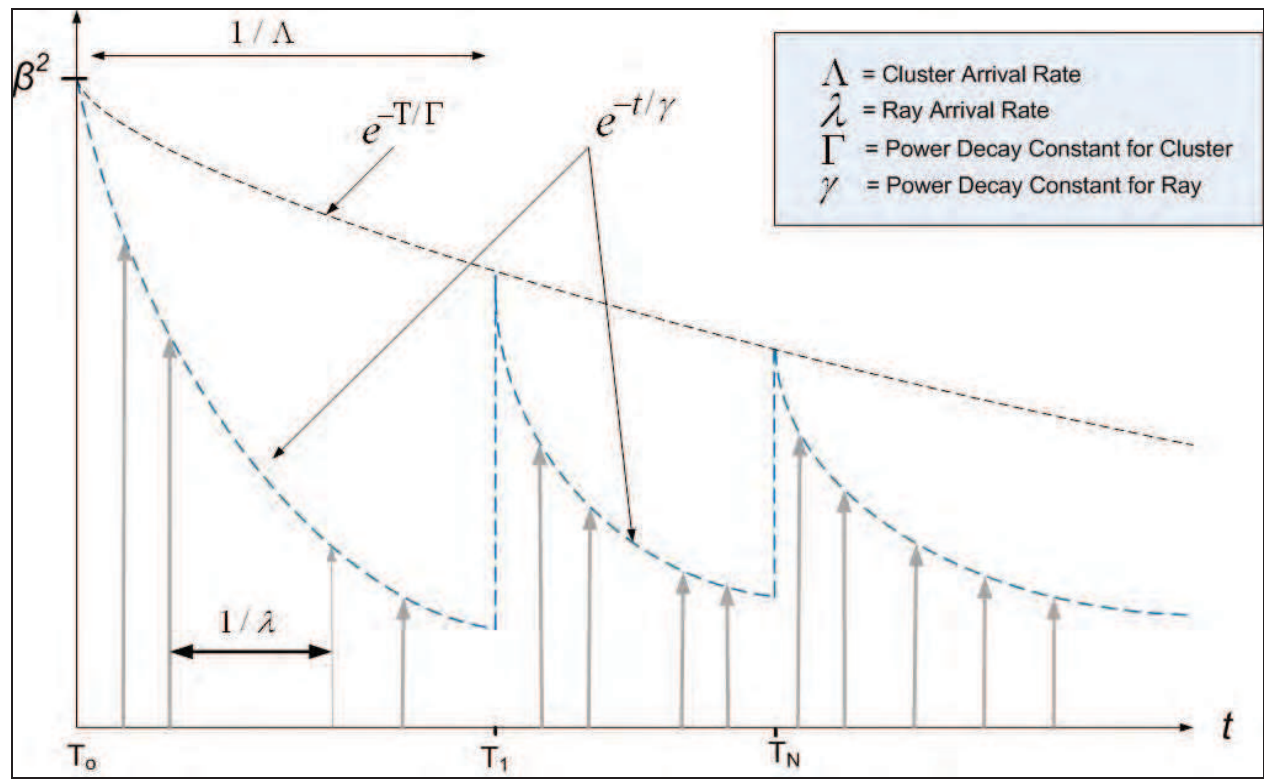

Fig. 21. Example channel impulse response derived from a Saleh and Valenzuela channel model. (After [A. A. M. Saleh et. al.].)

6. The MIMO channel matrix $\mathbf{H}$ for each filter tap at each instant of time is separated into a fixed (constant) LOS matrix, $\mathbf{H}_{\mathrm{F}}$, and a time-varying (Rayleigh distributed) NLOS matrix, $\mathbf{H}_{\mathbf{V}}$ [V. Erceg et. al., 2004], i.e.:

$$
\mathrm{H}=\left(\sqrt{\frac{K}{K+1}} \mathrm{H}_{\mathrm{F}}+\sqrt{\frac{1}{K+1}} \mathrm{H}_{\mathrm{V}}\right)
$$

where $K$ is the Ricean $K$-factor.

7. Each tap consists of a number of sub-paths having truncated Laplacian azimuth spectrum with an angular spread that varies between from $20^{\circ}$ to $40^{\circ}$ [V. Erceg et. al., 2004].

8. Elevation spread is not incorporated (since most building dimensions in azimuth are much larger than their dimensions in elevation) [V Erceg et. al., 2004]).

9. The mean DOA and DOD for each cluster is random with a uniform distribution over all azimuth angles. (For indoor WLANs the scattering environment is similar for both the access point and the user equipment. This is in contrast to outdoor scenarios where the BS is typically mounted sufficiently high to be relatively free of local scatter while the MS is often immersed in a rich scattering environment.)

10. Several channel taps in scenarios D and E are amplitude modulated to account for the effects of fluorescent lights [V. Erceg et. al., 2004] which represent time- 
periodic scatterers; alternately present and absent at twice the frequency of the mains supply $(2 \times 50 \mathrm{~Hz}$ in Europe).

11. The model differentiates between uplink and downlink (unlike SCM and SCME). 12. Isotropic antenna elements are assumed.

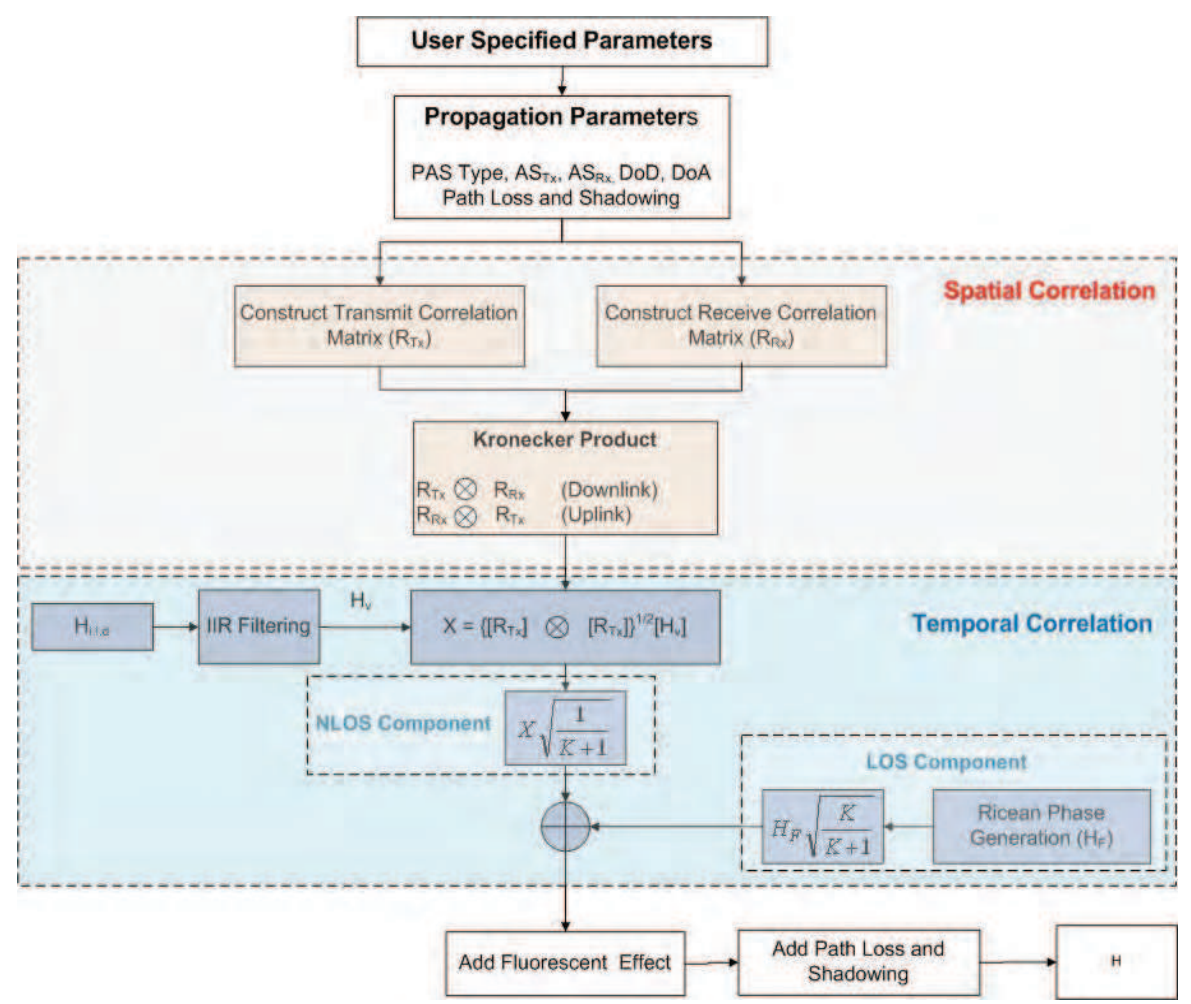

Fig. 22. IEEE $802.11 \mathrm{n}$ channel model

\subsubsection{Model features}

The various steps in the IEEE 802.11n channel model are illustrated in Fig. 22 and described below:

1. At each drop, during the initialisation phase, the model determines the delay profile and identifies clusters in the user-specified scenario.

2. The model assigns azimuth spread, mean DOD and DOA to each cluster and the corresponding taps. Spatial correlation is calculated independently at transmit and receive antennas and is based on antenna geometry.

3. The spatial correlation matrix is the Kronecker product of the individual spatial correlation matrices of transmit and receives antenna arrays, Fig. 22. The spatial correlation is a square matrix and is different for uplink and downlink. 
4. Fast fading samples are generated by filtering uncorrelated (white) Gaussian noise. The filter final states are retained after a dummy run in order to avoid transient states. (Fading samples generated during the transient phase are not used because their variance is artificially low.)

5. In order to maintain continuity between successive channel calls (for the same drop) the filter states are stored.

6. The product of spatial and temporal correlation matrices results in the $\mathbf{H}$ matrix which is then scaled to account for path-loss and shadowing.

\section{Summary and comparison of channel models}

Table 1 summarises the principal features of those channel models that have been described and Table 2 compares some of their most important parameters.

\begin{tabular}{|c|c|c|c|c|c|c|}
\hline & SCM & SCME & WINNER II & sul & WIMAX & IEEE $802.11 n$ \\
\hline Bandwidth $>100 \mathrm{MHz}$ & No & Yes $^{*}$ & Yes & No & No & Yes \\
\hline Indoor scenarios & No & No & Yes & No & No & Yes ** \\
\hline $\begin{array}{l}\text { Outdoor-to-indoor and } \\
\text { indoor-to-outdoor scenarios }\end{array}$ & No & No & Yes & No & No & No \\
\hline DoD/DoA elevation & No & No & Yes & No & No & No \\
\hline $\begin{array}{l}\text { Random selection of MPC from the } \\
\text { appropriate probability distribution }\end{array}$ & Yes & Yes & Yes & No & No & No \\
\hline $\begin{array}{l}\text { Time evolution of large scale model } \\
\text { parameters with in a drops }\end{array}$ & No & Yes & Yes & No & No & No \\
\hline $\begin{array}{l}\text { Time evolution of large scale model } \\
\text { parameters between successive drops }\end{array}$ & No & No & Yes & No & No & No \\
\hline $\begin{array}{l}\text { Use of Intra-Cluster delay spread concept } \\
\text { (based on Saleh and Valenzuala model }\end{array}$ & No & Yes & Yes & No & No & Yes \\
\hline $\begin{array}{l}\text { Cross correlation between large scale } \\
\text { parameters }\end{array}$ & No & No & Yes & No & No & No \\
\hline
\end{tabular}

* SCME suppot both $2 \mathrm{CMHz}$ and $10 \mathrm{C} \mathrm{MHz}$ banowititr.

* IEEE802.11ti is an indoor channel model with only one

Table 1. Principal features of standard channel models. (Extended from [M. Narandzic et. al., 2007].) 


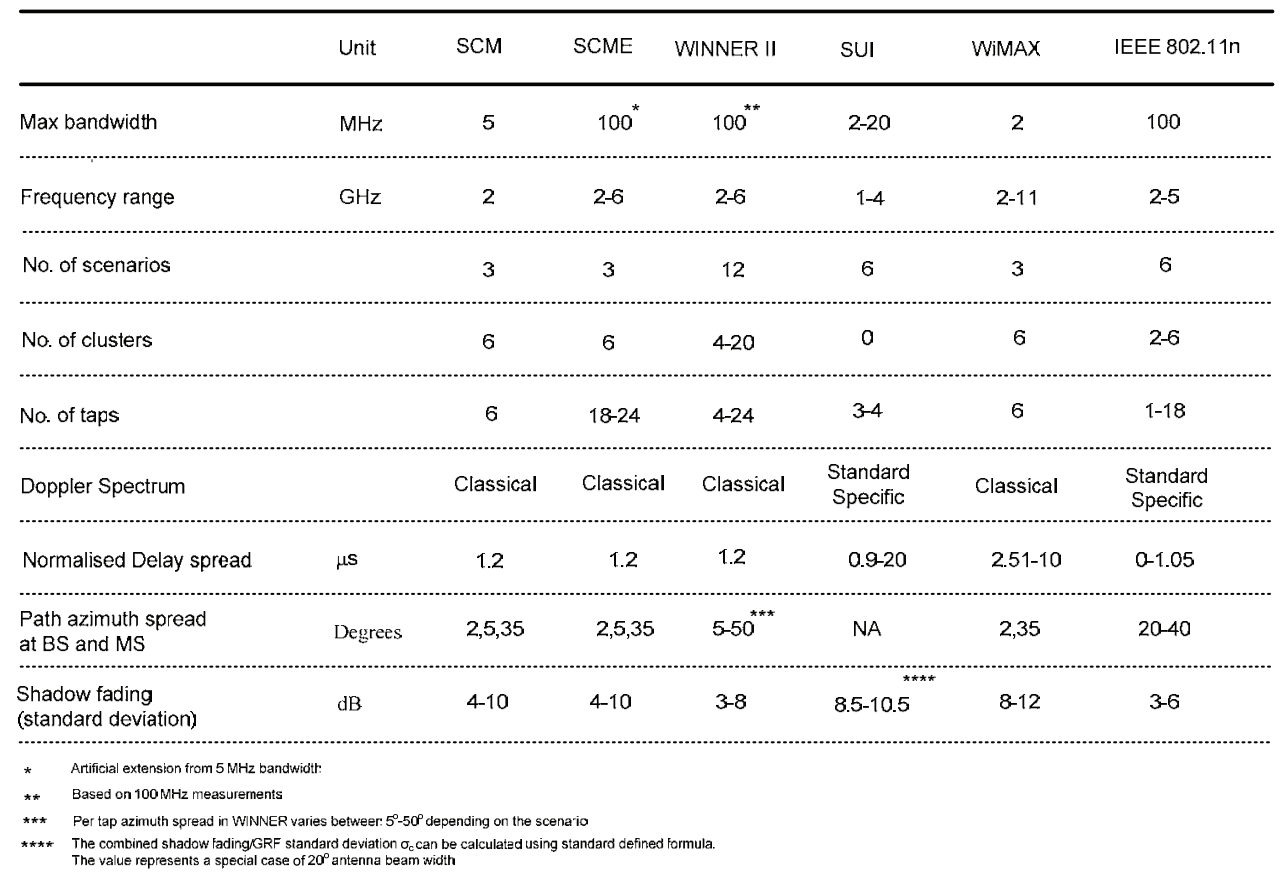

Table 2. Comparison of standard channel model parameters. (Extended from [M. Narandzic et. al., 2007].)

\section{Summary}

Next generation wireless systems will offer wide bandwidth, high data-rates and greater mobility. MIMO technology will certainly play an important role in future wireless application. This chapter has presented a brief review of the theoretical framework used to describe MIMO channels and has described a selection of standard MIMO channel models. The characteristics of standard channel models have been summarised and compared.

\section{Reference}

A.A.M. Saleh. \& R.A. Valenzuela (1987). "A statistical model for indoor multipath propagation," IEEE Journal on Selected Areas in Communications, vol. 5, 1987, pp. 128-137.

A.M. Sayeed, "Deconstructing multiantenna fading channels," IEEE Transactions on Signal Processing, vol. 50, no. 10, pp. 2563 - 2579, October 2002.

A. Burr, "Capacity bounds and estimates for the finite scatterers MIMO wireless channel," IEEE Journal on Selected Areas in Communications, vol. 21, no. 5, pp. 812-818, 2003.

A. C. Ludwig, "The definition of cross polarization, IEEE Transactions on Antennas and Propagation. AP-21(1) pp. 116-119, January 1973. 
P. Almers.; F. Tufvesson.; A.F. Molisch., "Keyhold Effect in MIMO Wireless Channels: Measurements and Theory", IEEE Transactions on Wireless Communications, ISSN: 1536-1276, Vol. 5, Issue 12, pp. 3596-3604, December 2006.

D.S. Baum.; j. Hansen.; j. Salo., "An interim channel model for beyond-3G systems: extending the 3GPP spatial channel model (SCM)," Vehicular Technology Conference, 2005. VTC 2005-Spring. 2005 IEEE 61st, vol.5, no., pp. 3132-3136 Vol. 5, 30 May-1 June 2005.

N. Czink.; A. Richter.; E. Bonek.; J.-P. Nuutinen.; j. Ylitalo., "Including Diffuse Multipath Parameters in MIMO Channel Models," Vehicular Technology Conference, 2007. VTC-2007 Fall. 2007 IEEE 66th, vol., no., pp.874-878, Sept. 30 2007-Oct. 32007.

D.-S. Shiu.; G. J. Foschini.; M. J. Gans.; and J. M. Kahn, “Fading correlation and its effect on the capacity of multielement antenna systems," IEEE Transactions on Communications, vol. 48, no. 3, pp. 502-513, 2000.

H. El-Sallabi.; D.S Baum.; P. ZetterbergP.; P. Kyosti.; T. Rautiainen.; C. Schneider., "Wideband Spatial Channel Model for MIMO Systems at $5 \mathrm{GHz}$ in Indoor and Outdoor Environments," Vehicular Technology Conference, 2006. VTC 2006Spring. IEEE 63rd , vol.6, no., pp.2916-2921, 7-10 May 2006.

E. Telatar, "Capacity of multi-antenna Gaussian channels," European Transactions on Telecommunications, vol. 10, no. 6, pp. 585-595, 1999.

E.T. Jaynes, "Information theory and statistical mechanics," APS Physical Review, vol. 106, no. 4, pp. 620-630, 1957.

3GPP TR25.996 V6.1.0 (2003-09) "Spatial channel model for multiple input multiple output (MIMO) simulations" Release 6. (3GPP TR 25.996)

IEEE 802.16 (BWA) Broadband wireless access working group, Channel model for fixed wireless applications, 2003. http://ieee802.org/16

IEEE 802.11, WiFi. http://en.wikipedia.org/wiki/IEEE_802.11-2007. Last assessed on 01May 2009.

International Telecommunications Union, "Guidelines for evaluation of radio transmission technologies for imt-2000," Tech. Rep. ITU-R M.1225, The International Telecommunications Union, Geneva, Switzerland, 1997

Jakes model; http://en.wikipedia.org/wiki/Rayleigh_fading

J. P. Kermoal.; L. Schumacher.; K. I. Pedersen.; P. E. Mogensen'; and F. Frederiksen, “A stochastic MIMO radio channel model with experimental validation," IEEE Journal on Selected Areas in Communications, vol. 20, no. 6, pp. 1211-1226, 2002.

J. W. Wallace and M. A. Jensen, "Modeling the indoor MIMO wireless channel," IEEE Transactions on Antennas and Propagation, vol. 50, no. 5, pp. 591-599, 2002.

L.J. Greenstein, S. Ghassemzadeh, V.Erceg, and D.G. Michelson, "Ricean K-factors in narrowband fixed wireless channels: Theory, experiments, and statistical models," WPMC'99 Conference Proceedings, Amsterdam, September 1999.

Merouane Debbah and Ralf R. M"uller, "MIMO channel modelling and the principle of maximum entropy," IEEE Transactions on Information Theory, vol. 51, no. 5, pp. 1667-1690, May 2005.

M. Steinbauer, "A Comprehensive Transmission and Channel Model for Directional Radio Channels," COST 259, No. TD(98)027. Bern, Switzerland, February 1998. 13. M. Steinbauer, "A Comprehensive Transmission and Channel Model for Directional Radio Channels," COST259, No. TD(98)027. Bern, Switzerland, February 1998. 
M. Steinbauer.; A. F. Molisch, and E. Bonek, "The doubledirectional radio channel," IEEE Antennas and Propagation Magazine, vol. 43, no. 4, pp. 51-63, 2001.

M. Narandzic.; C. Schneider .; R. Thoma.; T. Jamsa.; P. Kyosti.; Z. Xiongwen, "Comparison of SCM, SCME, and WINNER Channel Models," Vehicular Technology Conference, 2007. VTC2007-Spring. IEEE 65th, vol., no., pp.413-417, 22-25 April 2007.

M. Ozcelik.;N. Czink.; E. Bonek ., "What makes a good MIMO channel model?," Vehicular Technology Conference, 2005. VTC 2005-Spring. 2005 IEEE 61'st, vol.1, no., pp. 156160 Vol. 1, 30 May-1 June 2005.

P.Almer.; E.Bonek.; A.Burr.; N.Czink.; M.Deddah.; V.Degli-Esposti.; H.Hofstetter.; P.Kyosti.; D.Laurenson.; G.Matz.; A.F.Molisch.; C.Oestges and H.Ozcelik."Survey of Channel and Radio Propagation Models for Wireless MIMO Systems". EURASIP Journal on Wireless Communications and Networking, Volume 2007 (2007), Article ID 19070, 19 pages doi:10.1155/2007/19070.

Paul BS.; Bhattacharjee R. MIMO Channel Modeling: A Review. IETE Tech Rev 2008;25:315-9

Spirent Communications.; Path-Based Spatial Channel Modelling SCM/SCME white paper 102. 2008.

SCME Project; 3GPP Spatial Channel Model Extended (SCME); http://www.ist winner.org/3gpp_scme.html.

T. S. Rapport (2002). Wireless Communications Principles and Practice, ISBN 81-7808-648-4, Singapore.

T. Zwick.; C. Fischer, and W. Wiesbeck, "A stochastic multipath channelmodel including path directions for indoor environments,"IEEE Journal on Selected Areas in Communications, vol. 20, no. 6, pp. 1178-1192, 2002.

V Erceg.; L Schumacher.; P Kyristi.; A Molisch.; D S. Baum.; A Y Gorokhov.; C Oestges.; Q Li, K Yu.; N Tal, B Dijkstra.; A Jagannatham.; C Lanzl.; V J. Rhodes.; J Medos.; D Michelson.; M Webster.; E Jacobsen.; D Cheung.; C Prettie.; M Ho.; S Howard.; B Bjerke.; L Jengx.; H Sampath.; S Catreux.; S Valle.; A Poloni.; A Forenza.; R W Heath. "TGn Channel Model". IEEE P802.11 Wireless LANs. May 10, 2004. doc IEEE 802.11-03/940r4.

R. Verma.; S. Mahajan.; V. Rohila., "Classification of MIMO channel models," Networks, 2008. ICON 2008. $16^{\text {th }}$ IEEE International Conference on, vol., no., pp.1-4, 12-14 Dec. 2008.

WINNER.; Final Report on Link Level and System Level Channel Models. IST-2003-507581 WINNER. D5.4 v. 1.4, 2005.

WINNER II Channel Models. IST-4-027756 WINNER II D1.1.2 V1.1, 2007.

WINNER II interim channel models. IST-4-027756 WINNER II D1.1.1 V1.1, 2006.

S. Wyne.; A.F. Molisch.; P. Almers.; G. Eriksson.; J. Karedal.; F. Tufvesson., "Statistical evaluation of outdoor-to-indoor office MIMO measurements at $5.2 \mathrm{GHz}$," Vehicular Technology Conference, 2005. VTC 2005-Spring. 2005 IEEE 61st, vol.1, no., pp. 146150 Vol. 1, 30 May-1 June 2005

WiMAX forum ${ }^{\circledR}$. Mobile Release 1.0 Channel Model. 2008.

wikipedia.org. http:/ /en.wikipedia.org/wiki/IEEE_802.11n. Last assessed on May 2009. 


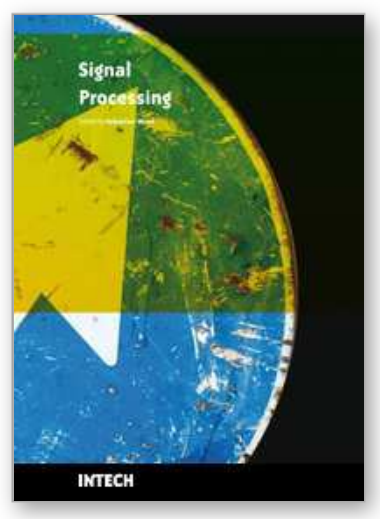

\author{
Signal Processing \\ Edited by Sebastian Miron
}

ISBN 978-953-7619-91-6

Hard cover, 528 pages

Publisher InTech

Published online 01, March, 2010

Published in print edition March, 2010

This book intends to provide highlights of the current research in signal processing area and to offer a snapshot of the recent advances in this field. This work is mainly destined to researchers in the signal processing related areas but it is also accessible to anyone with a scientific background desiring to have an up-to-date overview of this domain. The twenty-five chapters present methodological advances and recent applications of signal processing algorithms in various domains as telecommunications, array processing, biology, cryptography, image and speech processing. The methodologies illustrated in this book, such as sparse signal recovery, are hot topics in the signal processing community at this moment. The editor would like to thank all the authors for their excellent contributions in different areas of signal processing and hopes that this book will be of valuable help to the readers.

\title{
How to reference
}

In order to correctly reference this scholarly work, feel free to copy and paste the following:

Faisal Darbari, Robert W. Stewart and lan A. Glover (2010). MIMO Channel Modelling, Signal Processing, Sebastian Miron (Ed.), ISBN: 978-953-7619-91-6, InTech, Available from: http://www.intechopen.com/books/signal-processing/mimo-channel-modelling

\section{INTECH}

open science | open minds

\section{InTech Europe}

University Campus STeP Ri

Slavka Krautzeka 83/A

51000 Rijeka, Croatia

Phone: +385 (51) 770447

Fax: +385 (51) 686166

www.intechopen.com

\section{InTech China}

Unit 405, Office Block, Hotel Equatorial Shanghai

No.65, Yan An Road (West), Shanghai, 200040, China

中国上海市延安西路65号上海国际贵都大饭店办公楼 405 单元

Phone: +86-21-62489820

Fax: +86-21-62489821 
(C) 2010 The Author(s). Licensee IntechOpen. This chapter is distributed under the terms of the Creative Commons Attribution-NonCommercialShareAlike-3.0 License, which permits use, distribution and reproduction for non-commercial purposes, provided the original is properly cited and derivative works building on this content are distributed under the same license. 